\title{
Models of Systems Failure in Aging
}

\author{
Leonid A. Gavrilov and Natalia S. Gavrilova
}

\begin{abstract}
Mathematical models of systems failure are critically important for understanding the mechanisms of aging because aging of organisms is associated with increased risk of failure of its physiological systems.

Theoretical analysis of systems failure in aging leads naturally to apply the existing general theory of systems failure, which is also known as the reliability theory. Reliability-theory approach to biological aging is useful for three reasons. (1) It provides a useful scientific language (definitions and cross-cutting principles) helping to create a general theoretical framework for organizing numerous and diverse observation on aging into a coherent picture. This is very important for researchers, because it helps them to understand each other despite a disruptive specialization of aging studies. (2) It allows researchers to develop a scientific intuition and understanding of the main principles of the aging process by considering simplified mathematical models of systems failure, having some general features of real aging organisms. (3) It helps to generate and test specific predictions on age-related dynamics of systems failure, and to get deeper insights in the mechanisms of aging by more creative analysis of already collected data.

This chapter reviews the existing theoretical reliability models and approaches, which help us to understand the mechanisms and age-dynamics of systems failure. Empirical observations on systems failure in aging are also reviewed (the Gompertz and Weibull failure laws, the compensation law of systems failure, and the late-life failure rate leveling-off), and are theoretically explained through the observed decline in system's redundancy with age. The causes of failure rate increase with age are discussed and explained using five simple mathematical models of systems failure in aging as an illustration.
\end{abstract}

\section{Introduction}

This introductory-overview chapter sets a stage for introducing theoretical models of systems failure in aging. Mathematical models of systems failure are important for the studying of human aging, because aging is associated with increased risk of failure in human physiological systems. Theoretical analysis of systems failure in aging invites us to consider the general theory of systems failure known as reliability theory (Barlow and Proshan, 1975; Barlow et al., 1965; Gavrilov, 1978; Gavrilov and Gavrilova, 1991, 2001, 2003a, 2004a, 2005; Gavrilov et al., 1978).

Reliability theory was historically developed to describe failure and aging of complex electronic (military) equipment, but the theory itself is a very general theory based on mathematics (probability theory) and systems approach (Barlow and Proschan, 1975; Barlow et al., 1965). It may therefore be useful to describe and understand the aging and failure of biological systems too. Reliability theory may be useful in several ways: first, by providing a kind of scientific language (definitions and cross-cutting principles), which helps to create a logical framework for organizing numerous and diverse observations on aging into a coherent picture. Second, it helps researchers to develop an intuition and an understanding of the main principles of the aging process through consideration of simple mathematical models, having some features of a real world. Third, reliability theory is useful for generating and testing specific predictions, as well as deeper analyses of already collected data. The purpose of this chapter is to review the theoretical reliability models and approaches, which help to understand the mechanisms and dynamics of systems failure in aging.

\section{Reliability Approach to System's Failure in Aging}

Reliability theory is a body of ideas, mathematical models, and methods directed to predict, estimate, understand, and optimize the lifespan and failure distributions of systems and their components (adapted from Barlow and Proschan, 1975). Reliability theory allows researchers to predict the age-related failure kinetics for a system of given architecture (reliability structure) and given reliability of its components.

\section{DEFINITIONS OF SYSTEM'S FAILURE AND AGING}

The concept of failure is important to the analysis of system's reliability. In reliability theory failure is defined as the event when a required function is terminated (Rausand and Houland, 2003). In other words, failure is such an outcome when the system deviates from 
optimistically anticipated and desired behavior ("fails"). Failures are often classified into two groups:

1. degradation failures, where the system or component no longer functions properly, and

2. catastrophic or fatal failures - the end of system's or component's life.

Good examples of degradation failures in humans would be an onset of different types of health impairments, diseases, or disabilities, while catastrophic or fatal failures obviously correspond to death. The notions of aging and failure are related to each other in the following way: when the risk of failure outcomes increases with age ("old is not as good as new") - this is aging by definition. Note that according to reliability theory, aging is not just growing old; instead aging is a degradation leading to failure (adverse health outcomes)-becoming sick, disabled, frail and dead. Therefore, from a reliabilitytheory perspective the notion of "healthy aging" is an oxymoron like a healthy dying, or a healthy disease. More appropriate terms instead of "healthy aging" or "aging well" would be a delayed aging, postponed aging, slow aging, arrested aging, or negligible aging (senescence).

Because the reliability definition of biological aging is linked to health failures (adverse health outcomes, including death), aging without diseases is just as inconceivable as dying without death. Diseases and disabilities are an integral part (outcomes) of the aging process. Not every disease is related to aging, but every progression of disease with age has some relevance to aging: aging is a "maturation" of diseases with age.

Note that a system may have an aging behavior for one particular type of failure, but it may remain to be as good as new for some other type of failure. Thus the notion of aging is outcome-specific - it requires specifying for which particular type of failure (or group of failures) the system deteriorates. Consequently, the legitimate antiaging interventions may be outcome-specific too, and limited to postponing some specific adverse health outcomes. Aging is likely to be a summary term for many different processes leading to various types of degradation failures, and each of these processes deserves to be studied and prevented.

\section{BASIC FAILURE MODELS}

Reliability of the system (or its component) refers to its ability to operate properly according to a specified standard (Crowder et al., 1991). Reliability is described by the reliability function $S(x)$, which is the probability that a system (or component) will carry out its mission through time $x$ (Rigdon and Basu, 2000). The reliability function (also called the survival function) evaluated at time $x$ is just the probability $P$, that the failure time $X$, is beyond time $x$. Thus, the reliability function is defined in the following way:

$$
S(x)=P(X>x)=1-P(X \leq x)=1-F(x)
$$

where $F(x)$ is a standard cumulative distribution function in the probability theory (Feller, 1968). The best illustration for the reliability function $S(x)$ is a survival curve describing the proportion of those still alive by time $x$ (the $l_{x}$ column in life tables).

Failure rate, $\mu(x)$, or instantaneous risk of failure, also called the hazard rate, $h(x)$, or mortality force is defined as the relative rate for reliability function decline:

$$
\mu(x)=-\frac{d S_{x}}{S_{x} d x}=\frac{-d \ln S_{x}}{d x}
$$

In those cases when the failure rate is constant (does not increase with age), we have nonaging system (component) that does not deteriorate (does not fail more often) with age:

$$
\mu(x)=\mu=\mathrm{const}
$$

The reliability function of nonaging systems (components) is described by the exponential distribution:

$$
S(x)=S_{0} e^{-\mu x}
$$

This failure law describes "lifespan" distribution of atoms of radioactive elements, and, therefore, it is often called an exponential decay law. Interestingly, this failure law is observed in many wild populations with high extrinsic mortality (Finch, 1990; Gavrilov and Gavrilova, 1991). This kind of distribution is observed if failure (death) occurs entirely by chance, and it is also called a "one-hit model," or a "first order kinetics." The nonaging behavior of a system can be detected graphically when the logarithm of the survival function decreases with age in a linear fashion.

Recent studies found that at least some cells in the aging organism might demonstrate nonaging behavior. Specifically, the rate of neuronal death does not increase with age in a broad spectrum of aging-related neurodegenerative conditions (Heintz, 2000). These include 12 different models of photoreceptor degeneration, "excitotoxic" cell death in vitro, loss of cerebellar granule cells in a mouse model, and Parkinson's and Huntington's diseases (Clarke et al., 2000). In this range of diseases, five different neuronal types are affected. In each of these cases, the rate of cell death is best fit by an exponential decay law with constant risk of death independent of age (death by chance only), arguing against models of progressive cell deterioration and aging (Clarke et al., 2000, 2001a). An apparent lack of cell aging is also observed in the case of amyotrophic lateral sclerosis (Clarke et al., 2001a), retinitis pigmentosa (Burns, 2002; Clarke et al., 2000, 2001a; Massof, 1990) and idiopathic Parkinsonism (Calne, 1994; Clarke et al., 2001b; Schulzer et al., 1994). These observations correspond well with another observation that "an impressive range of cell functions in most organs remain unimpaired throughout the life span" (Finch, 1990, p. 425), and these unimpaired functions might reflect the "no-aging" property known as "old as good as new" property in survival 


\section{Models of Systems Failure in Aging}

analysis (Klein and Moeschberger, 1997, p. 38). Thus we come to the following fundamental question about the origin of aging: How can we explain the aging of a system built of nonaging elements? This question invites us to think about the possible systemic nature of aging and to wonder whether aging may be a property of the system as a whole. We would like to emphasize the importance of looking at the bigger picture of the aging phenomenon in addition to its tiny details, and we will suggest a possible answer to the posed question in this chapter.

If failure rate increases with age, we have an aging system (component) that deteriorates (fails more often) with age. There are many failure laws for aging systems, and the most famous one in biology is the Gompertz law with exponential increase of the failure rates with age (Finch, 1990; Gavrilov and Gavrilova, 1991; Gompertz, 1825; Makeham, 1860; Strehler, 1978):

$$
\mu(x)=R e^{\alpha x}
$$

An exponential (Gompertzian) increase in death rates with age is observed for many biological species including fruit flies Drosophila melanogaster (Gavrilov and Gavrilova, 1991), nematodes (Brooks et al., 1994; Johnson, 1987, 1990), mosquitoes (Gavrilov, 1980), human lice, Pediculus humanus (Gavrilov and Gavrilova, 1991), flour beetles, Tribolium confusum (Gavrilov and Gavrilova, 1991), mice (Kunstyr and Leuenberger, 1975; Sacher, 1977), rats (Gavrilov and Gavrilova, 1991), dogs (Sacher, 1977), horses (Strehler, 1978), mountain sheep (Gavrilov, 1980), baboons (Bronikowski et al., 2002) and, perhaps most important, humans (Finch, 1990; Gavrilov and Gavrilova, 1991; Gompertz, 1825; Makeham, 1860; Strehler, 1978). According to the Gompertz law, the logarithm of failure rates increases linearly with age. This is often used in order to illustrate graphically the validity of the Gompertz law - the data are plotted in the semilog scale (known as the Gompertz plot) to check whether the logarithm of the failure rate is indeed increasing with age in a linear fashion.

For technical systems one of the most popular models for failure rate of aging systems is the Weibull model, the power-function increase in failure rates with age (Weibull, 1939):

$$
\mu(x)=\alpha x^{\beta} \text { for } x \geq 0, \quad \text { where } \alpha, \beta>0
$$

This law was suggested by Swedish engineer and mathematician W. Weibull in 1939 to describe the strength of materials (Weibull, 1939). It is widely used to describe aging and failure of technical devices (Barlow and Proschan, 1975; Rigdon and Basu, 2000; Weibull, 1951), and occasionally it was also applied to a limited number of biological species (Eakin et al., 1995; Hirsch and Peretz, 1984; Hirsch et al., 1994; Janse et al., 1988; Ricklefs and Scheuerlein, 2002; Vanfleteren et al., 1998). According to the Weibull law, the logarithm of failure rate increases linearly with the logarithm of age with a slope coefficient equal to parameter $\beta$. This is often used in order to illustrate graphically the validity of the Weibull law - the data are plotted in the log-log scale (known as the Weibull plot) to check whether the logarithm of the failure rate is indeed increasing with the logarithm of age in a linear fashion.

We will show later that both the Gompertz and the Weibull failure laws have a fundamental explanation rooted in reliability theory. Therefore it may be interesting and useful to compare these two failure laws and their behavior.

Figure 5.1a presents the dependence of the logarithm of the failure rate on age (Gompertz plot) for the Gompertz and the Weibull functions.

Note that in Figure 5.1a this dependence is strictly linear for the Gompertz function (as expected), and concave-down for the Weibull function. So the Weibull function looks like decelerating with age if compared to the Gompertz function.

Figure $5.1 \mathrm{~b}$ presents the dependence of the logarithm of the failure rate on the logarithm of age (Weibull plot) for the Gompertz and the Weibull functions. Note that this dependence is strictly linear for the Weibull function (as expected), and concave-up for the Gompertz function. So the Gompertz function looks like the accelerating one with the logarithm of age if compared to the Weibull function.

There are two fundamental differences between the Weibull and the Gompertz functions.

First, the Weibull function states that the system is immortal at starting age - when the age $X$ is equal to zero, the failure rate is equal to zero too, according to the Weibull formula. This means that the system should be initially ideal (immortal) in order for the Weibull law to be applicable to it. On the contrary, the Gompertz function states that the system is already vulnerable to failure at starting age - when the age $X$ is equal to zero, the failure rate is already above zero, equal to parameter $\mathrm{R}$ in the Gompertz formula. This means that the partially damaged systems having some initial damage load are more likely to follow the Gompertz failure law, while the initially perfect systems are more likely to follow the Weibull law.

Second, there is a fundamental difference between the Gompertz and the Weibull functions regarding their response to misspecification of the starting age ("age zero"). This is an important issue, because in biology there is an ambiguity regarding the choice of a "true" age, when aging starts. Legally, it is the moment of birth, which serves as a starting moment for age calculation. However, from a biological perspective there are reasons to consider a starting age as a date either well before the birth date (the moment of conception in genetics, or a critical month of pregnancy in embryology), or long after the birth date (the moment of maturity, when the formation of a body is finally completed). This uncertainty in starting age has very different implications for data analysis with the Gompertz or the Weibull functions. 
Leonid A. Gavrilov and Natalia S. Gavrilova
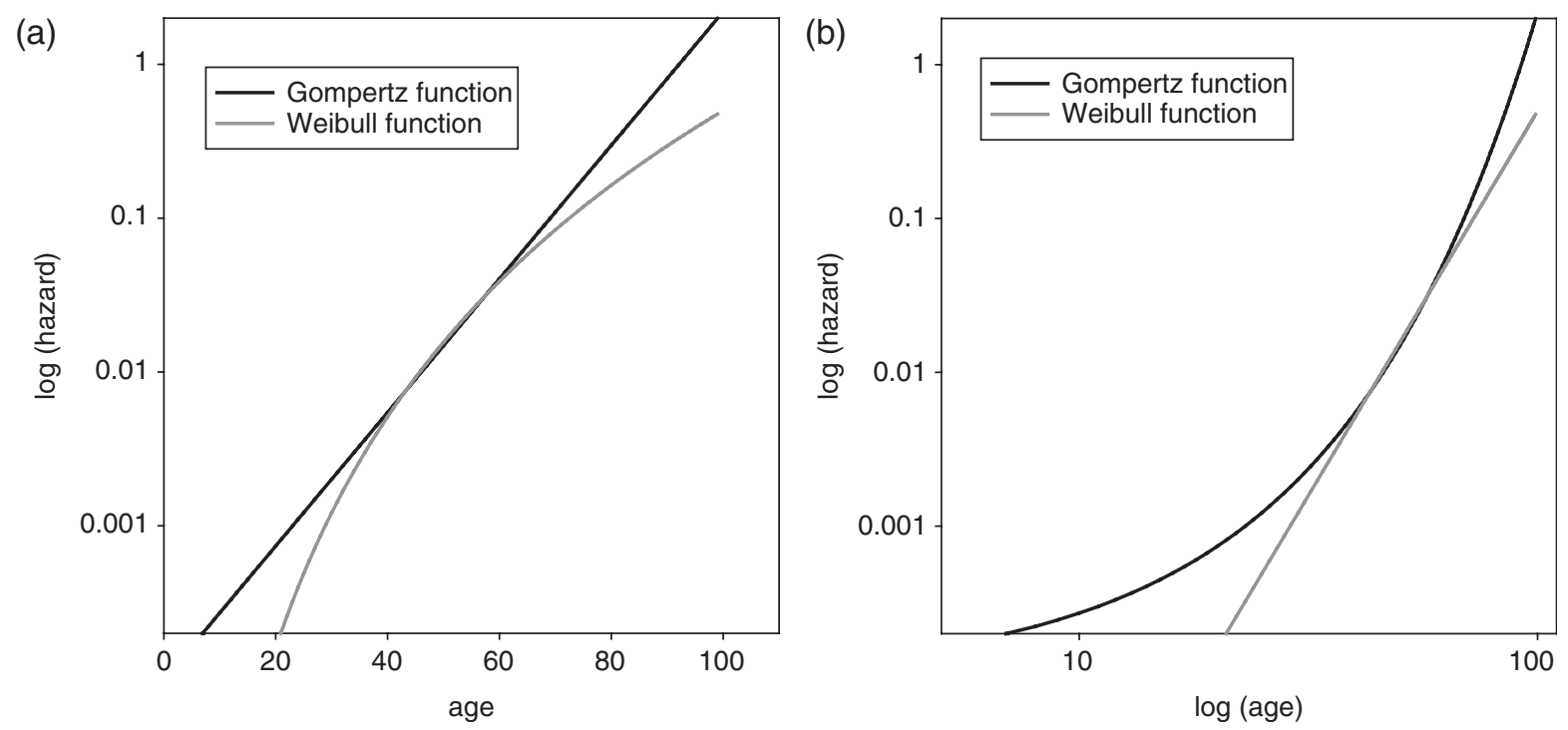

Figure 5.1. Plots of Gompertz and Weibull functions in different coordinates. (a) semilog (Gompertz) coordinates, (b) log-log (Weibull) coordinates. Source: Gavrilov and Gavrilova, 2005.

For the Gompertz function a misspecification of a starting age is not as important, because the shift in the age scale will still produce the same Gompertz function with the same slope parameter $\alpha$. The data generated by the Gompertz function with different age shifts will all be linear and parallel to each other in the Gompertz plot. The situation is very different for the Weibull function - it is linear in the Weibull plot for only one particular starting age, and any shifts in a starting age produce a different function. Specifically, if a "true" starting age is larger than assumed, then the resulting function will be a nonlinear concave-up curve in the Weibull plot indicating model misspecification and leading to a bias in estimated parameters. Thus, researchers choosing the Weibull function for data analysis have first to resolve an uneasy biological problem - at what age does aging start?

An alternative graceful mathematical solution of this problem would be to move from a standard twoparameter Weibull function to a more general threeparameter Weibull function, which has an additional "location parameter" $\gamma$ (Clark, 1975):

$\mu(x)=\alpha(x-\gamma)^{\beta}, \quad x>\gamma$, and equal to zero otherwise

Parameters of this formula, including the location parameter $\gamma$, could be estimated from the data through standard fitting procedures, thus providing a computational answer to a question "when does aging start?" However, this computational answer might be shocking to researchers, unless they are familiar with the concept of initial damage load, which is discussed elsewhere (Gavrilov and Gavrilova, 1991; 2001; 2004b; 2005).

In addition to the Gompertz and the standard twoparameter Weibull laws, a more general failure law was also suggested and theoretically justified using the systems reliability theory. This law is known as the binomial failure law (Gavrilov and Gavrilova, 1991; 2001; 2005), and it represents a special case of the three-parameter Weibull function with a negative location parameter:

$$
\mu(x)=\alpha\left(x_{0}+x\right)^{\beta}
$$

The parameter $x_{0}$ in this formula is called the initial virtual age of the system, IVAS (Gavrilov and Gavrilova, 1991; 2001; 2005). This parameter has the dimension of time and corresponds to the age by which an initially ideal system would have accumulated as many defects as a real system already has at the starting age (at $x=0$ ). In particular, when the system is initially undamaged, the initial virtual age of the system is zero and the failure rate grows as a power function of age (the Weibull law). However, as the initial damage load is increasing, the failure kinetics starts to deviate from the Weibull law, and eventually it evolves to the Gompertz failure law at high levels of initial damage load. This is illustrated in Figure 5.2, which represents the Gompertz plot for the data generated by the binomial failure law with different levels of initial damage load (expressed in the units of initial virtual age).

Note that as the initial damage load increases the failure kinetics evolves from the concave-down curves typical to the Weibull function, to an almost linear dependence between the logarithm of failure rate and age (the Gompertz function). Thus, biological species dying according to the Gompertz law may have a high initial damage load, presumably because of developmental noise, and a clonal expansion of mutations occurred in the early development (Gavrilov and Gavrilova, 1991; 2001; 2003a; 2004b). 


\section{Models of Systems Failure in Aging}

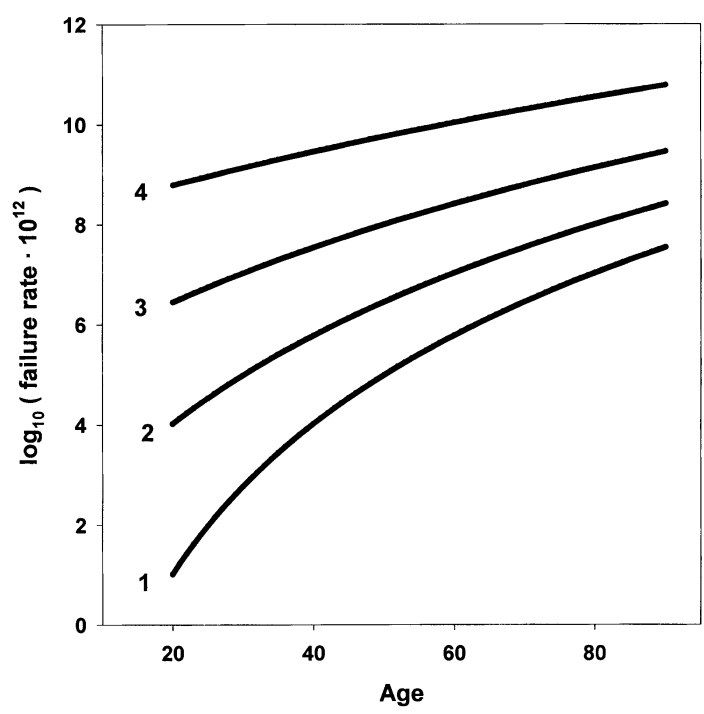

Figure 5.2. Failure kinetics of systems with different levels of initial damage. Dependence 1 is for initially ideal system (with no damage load). Dependence 2 is for system with initial damage load equivalent to damage accumulated by 20 -year-old system. Dependencies 3 and 4 are for systems with initial damage load equivalent to damage accumulated respectively by 50 -year-old and 100-year-old system. Source: Gavrilov and Gavrilova, 2005.

\section{SYSTEM'S FAILURE AND RELIABILITY STRUCTURE}

A branch of reliability theory, which studies reliability of an entire system given the reliability of its components and components' arrangement (reliability structure), is called system reliability theory (Rausand and Hoyland, 2003). System reliability involves the study of the overall performance of systems of interconnected components. The main objective of system reliability is the construction of a model that represents the times-to-failure of the entire system based on the life distributions of the components, from which it is composed. Consideration of some basic ideas and models of the system reliability theory is important because living organisms may be represented as structured systems comprised of organs, tissues and cells.

System reliability theory tells us that the component arrangement strongly affects the reliability of the whole system. The arrangement of components that are important for system reliability is also called reliability structure and is graphically represented by a schema of logical connectivity. It is important to understand that the model of logical connectivity is focused only on those components that are relevant for the functioning ability of the system. Components that do not play a direct role in the system's reliability usually are not included in the analyzed reliability structure (Rausand and Hoyland, 2003). For example, organs of vision are not included in the reliability structure of living organisms if death is the only type of failure to be analyzed (complete failure of vision does not cause an immediate death of the organism). On the other hand, if disability is the type of failure under consideration, then organs of vision should be included in the schema of reliability structure. Therefore, reliability structure does not necessarily reflect a physical structure of the object.

There are two major types of components arrangement (connection) in the system: components connected in series and components connected in parallel (Rausand and Hoyland, 2003). Here we consider a simple system of $n$ statistically independent components, where failure of one component does not affect the failure rate of other components of the system.

\section{Components connected in series}

For a system of $n$ independent components connected in series, the system fails if any one of the components fails, as in electrical circuits connected in series. Thus, failure of any one component results in failure of the whole system as in the Christmas tree lighting chains. Figure 5.3a shows a schema of the logical connectivity of the system in series.

This type of system is also called a weakest-link system (Ayyub and McCuen, 2003). In living organisms many organs and tissues (heart, lung, liver, brain) are vital for the organism's survival - a good example of series-connected components. Thus, the series connection means a logical connectivity, but not necessarily a physical or anatomical one.

The reliability of a system in series (with independent failure events of the components), $P_{\mathrm{s}}$, is a product of reliabilities of its components:

$$
P_{\mathrm{s}}=p_{1} p_{2} \ldots p_{n}
$$

where $p_{1} \ldots p_{n}$ are reliabilities of the system's components. This formula explains why complex systems with many critical components are so sensitive to early failures of their components.

For example, for a system built of 458 critical components, the initial period of the components' life when their cumulative risk of failure is only $1 \%$ corresponds to the end of the system's life when $99 \%$ of systems have already failed. This difference between the lifetimes of systems and the potential lifetimes of their components is increasing further with system complexity (numbers of critical components). Therefore, the early failure kinetics of components is so important in determining the failure kinetics of a complex system for its entire life.

The failure rate of a system connected in series is the sum of failure rates of its components (Barlow et al., 1965):

$$
\mu_{\mathrm{s}}=\mu_{1}+\mu_{2}+\mu_{i} \cdots+\mu_{n}
$$

If the failure rates of all components are equal, then the failure rate of the system with $n$ components is $n \mu$. It follows from this formula that if the system's 
Leonid A. Gavrilov and Natalia S. Gavrilova

(a)

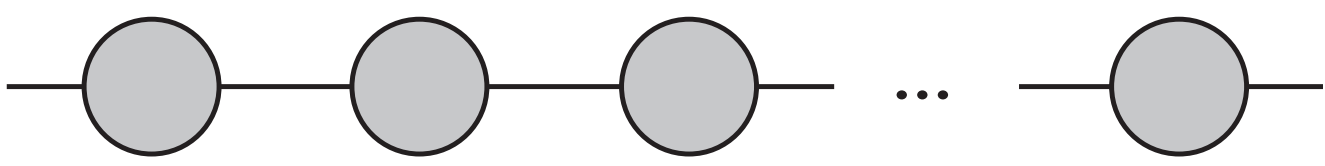

(b)

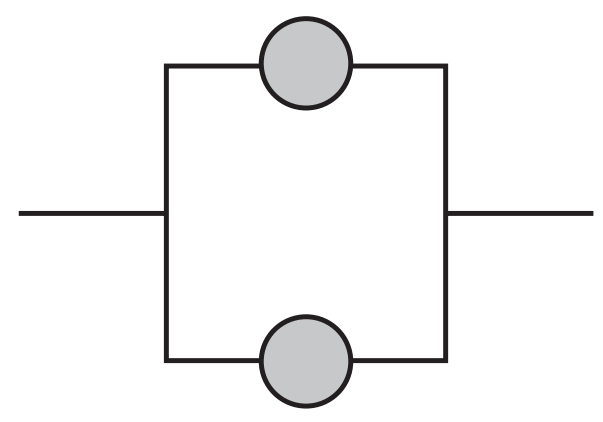

(c)

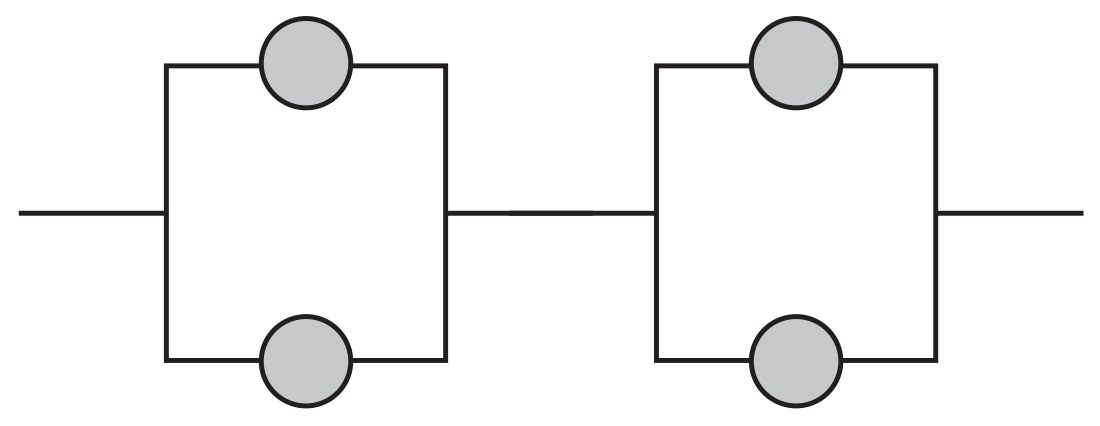

(d)

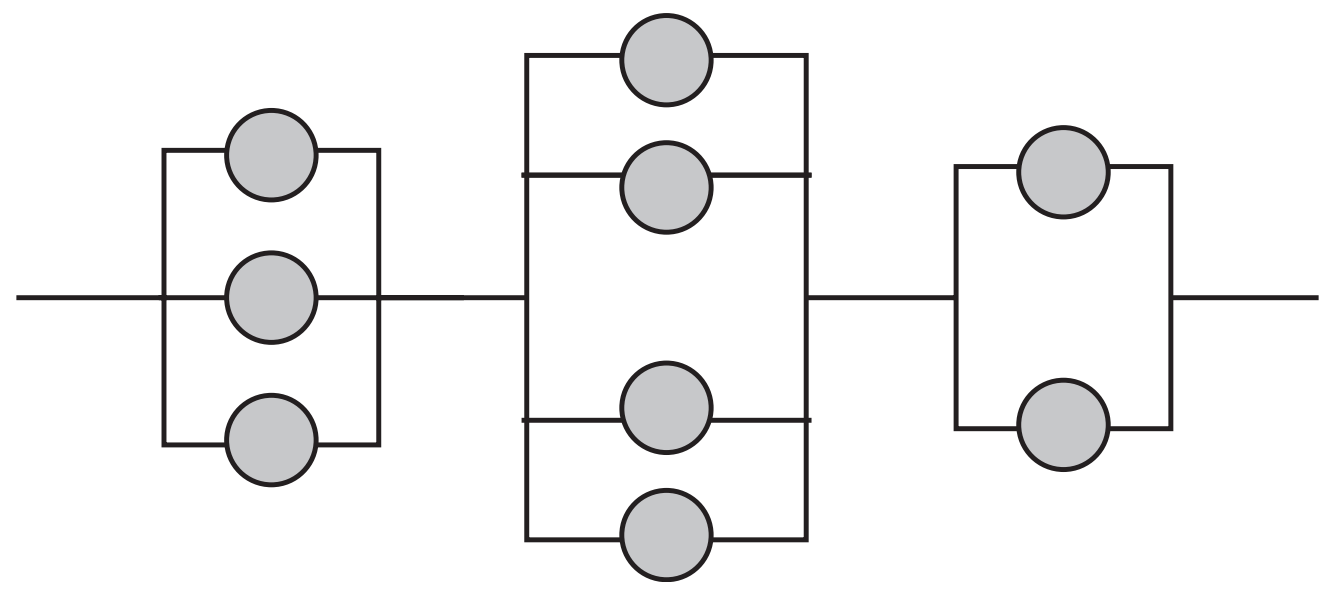

Figure 5.3. Logical schemas of systems with different types of elements connectivity: (a) system connected in series, (b) system connected in parallel, (c) series-parallel system, (d) series-parallel system with distributed redundancy, Source: Gavrilov and Gavrilova, 2005.

components do not age ( $\mu_{i}=$ const), then the entire system connected in series does not age either.

\section{Components connected in parallel}

A parallel system of a $n$ independent components fails only when all the components fail (as in electrical circuits connected in parallel). The logical structure of parallel system is presented in Figure 5.3b.
An example of a parallel system is a system with components all performing an identical function. This function will be destroyed only in the case when all the components fail. The number of additional components in parallel structure with one and the same function is called the redundancy or reserve of the system. In living organisms vital organs and tissues (such as liver, kidney, or pancreas) consist of many cells performing one and the same specialized function. 


\section{Models of Systems Failure in Aging}

For a parallel system with $n$ independent components the probability of the system's failure, $Q_{\mathrm{s}}$, is the product of the probabilities of failure for its components, $q_{i}$ :

$$
Q_{\mathrm{s}}=q_{1} q_{2} \ldots q_{n}
$$

Hence the reliability of a parallel system, $P_{\mathrm{s}}$, is related to the reliabilities of its components in the following way:

$$
P_{\mathrm{s}}=1-Q_{\mathrm{s}}=1-\left(1-p_{1}\right)\left(1-p_{2}\right) \ldots\left(1-p_{n}\right)
$$

The reliability of a parallel system with components of equal reliability, $p$, is

$$
P_{\mathrm{s}}=1-(1-p)^{n}
$$

What is very important is the emergence of aging in parallel systems - a parallel system is aging even if it is built of nonaging components with a constant failure rate (see more details in the section on causes of failure rate increase with age).

In a real world most systems are more complex than simply series and parallel structures, but in many cases they can be represented as combinations of these structures.

\section{More complex types of reliability structure}

The simplest combination of the two reliability structures is a series-parallel system with equal redundancy shown in Figure 5.3c.

A general series-parallel system is a system of $m$ subsystems (blocks) connected in series, where each block is a set of $n$ components connected in parallel. It turns out that even if the components themselves are not aging, the system as a whole has an aging behaviorits failure rate grows with age according to the Weibull law and then levels off at advanced ages (Gavrilov and Gavrilova, 1991; 2001, 2003a). This type of system is important to consider, because a living organism can be presented as a system of critical organs and tissues connected in series, while each organ consists of specialized cells connected in parallel. A reliability model for this type of system is described in more detail in the section on causes of failure rate increase with age.

Another type of reliability structure, a series-parallel system with distributed redundancy, was introduced by Gavrilov and Gavrilova in 1991 (Gavrilov and Gavrilova, 1991; 2001). The series-connected blocks of this system have nonequal redundancy (different numbers of elements connected in parallel), and the elements are distributed between the system's blocks according to some particular distribution law (see schema in Figure 5.3d).

Gavrilov and Gavrilova (1991; 2001) studied the reliability and failure rate of series-parallel systems with distributed redundancy for two special cases: (1) the redundancy distributed within an organism according to the Poisson law or (2) according to the binomial law. They found that the failure rate of such systems initially grows according to the Gompertz law (in the case of the Poisson distributed redundancy) or binomial failure law in the case of the binomially distributed redundancy (Gavrilov and Gavrilova, 1991; 2001). At advanced ages the failure rate for both systems asymptotically approaches an upper limit (mortality plateau). Reliability models for this type of system are described in more detail in the section on theoretical models of systems failure in aging.

Now that the basic concepts of reliability theory have been discussed, we may proceed to linking them to empirical observations on aging and mortality.

\section{Empirical Observations on Systems Failure in Aging}

\section{GENERAL OVERVIEW OF FAILURE KINETICS}

There is a striking similarity between living organisms and technical devices in the general age pattern of their failures - in both cases the failure rate usually follows the so-called bathtub curve (Figure 5.4).

The bathtub curve of failure rate is a classic concept presented in all textbooks on reliability theory (Ayyub and McCuen, 2003; Barlow and Proshan, 1975; Rausand and Hoyland, 2003).

The bathtub curve consists of three periods. Initially the failure rates are high and decrease with age. This period is called the "working-in" period and the period of "burning-out" of defective components. For example, the

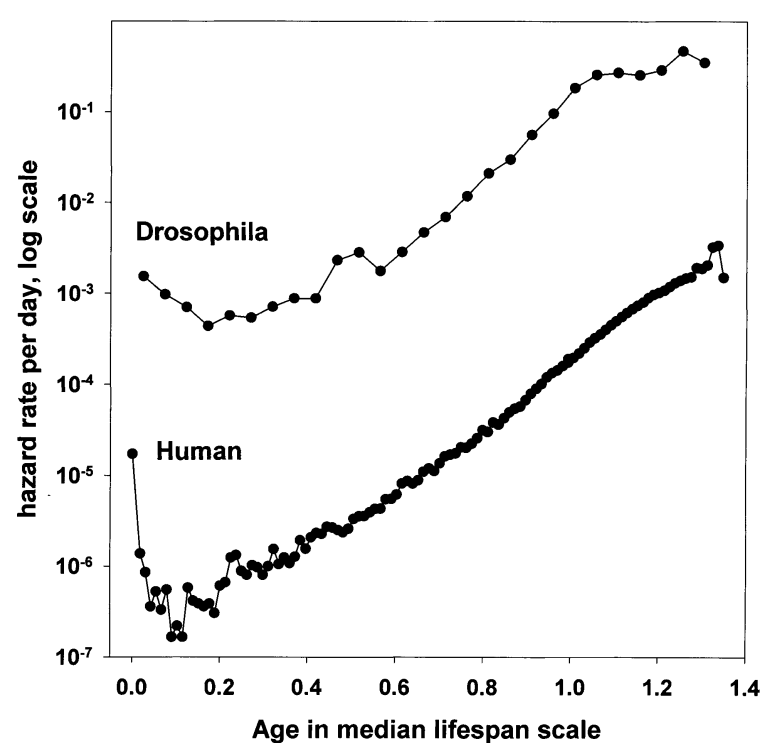

Figure 5.4. Bathtub mortality curves for humans and fruit flies Mortality is estimated on a daily basis; age is expressed in a median lifespan scale (a similar approach was used by Pearl and Miner, 1935 and Carnes et al., 1998). Mortality for Drosophila melanogaster was calculated using data published by Hall (1969). Mortality for humans was calculated using the official Swedish female life table for 1985 (ages 0-80 years), and the 1980-90 decennial life table for Swedish females available in the KannistoThatcher Database on Old Age Mortality, http://www.demogr. mpg.de/databases/ktdb (ages over 80 years), Source: Gavrilov and Gavrilova, 2005. 
risk for a new computer to fail is often higher at the very start, but then those computers that did not fail initially work normally afterwards. The same period exists early in life for most living organisms, including humans, and it is called the "infant mortality" period. Then follows the second period called "the normal working period," corresponding to an age of low and approximately constant failure rates. This period also exists in humans, but unfortunately it is rather short (10-15 years) and ends too soon. Then the third period, "the aging period," starts, which involves an inexorable rise in the failure rate with age. In most living organisms, including humans, this rise in failure rates follows an explosive exponential trajectory (the Gompertz curve). For humans, the aging period lies approximately within the interval 20-100 years. Thus there is a remarkable similarity in the failure patterns of technical and biological systems. This similarity is reinforced further by the fact that at extreme old ages there is one more, the fourth period common to both technical devices and living organisms (Economos, 1979). This period is known in biology as a period of late-life mortality leveling-off (Carey and Liedo, 1995; Clark and Guadalupe, 1995; Economos, 1979; Fukui et al., 1993; 1996; Vaupel et al., 1998), and also as the late-life mortality deceleration law (Fukui et al., 1993; 1996; Khazaeli et al., 1996; Partridge and Mangel, 1999).

\section{FAILURE LAWS IN SURVIVAL STUDIES}

Attempts to develop a fundamental quantitative theory of aging, mortality, and lifespan have deep historical roots. In 1825, the British actuary Benjamin Gompertz discovered a law of mortality (Gompertz, 1825), known today as the Gompertz law (Finch, 1990; Gavrilov and Gavrilova, 1991; Olshansky and Carnes, 1997; Strehler, 1978). Specifically, he found that the force of mortality increases in geometrical progression with the age of adult humans. According to the Gompertz law, human mortality rates double about every 8 years of adult age.

Gompertz also proposed the first mathematical model to explain the exponential increase in mortality rate with age (Gompertz, 1825). In reality, system failure rates may contain both nonaging and aging terms as, for example, in the case of the Gompertz-Makeham law of mortality (Finch, 1990; Gavrilov and Gavrilova, 1991; Makeham, 1860; Strehler, 1978):

$$
\mu(x)=A+R e^{\alpha x}
$$

In this formula the first, age-independent term (Makeham parameter, $A$ ) designates the constant, "nonaging" component of the failure rate (presumably due to external causes of death, such as accidents and acute infections), while the second, age-dependent term (the Gompertz function, $R e^{\alpha x}$ ) designates the "aging" component, presumably due to deaths from age-related degenerative diseases like cancer and heart disease.
The validity of the Gompertz-Makeham law of mortality can be illustrated graphically, when the logarithms of death rates without the Makeham parameter $\left(\mu_{x}-A\right)$ are increasing with age in a linear fashion. The log-linear increase in death rates (adjusted for the Makeham term) with age is indeed a very common phenomenon for many human populations at ages 35-70 years (Gavrilov and Gavrilova, 1991).

Note that the slope coefficient $\alpha$ characterizes an "apparent aging rate" (how rapid is the age-deterioration in mortality) - if $\alpha$ is equal to zero, there is no apparent aging (death rates do not increase with age).

At advanced ages (after age 70), the "old-age mortality deceleration" takes place - death rates are increasing with age at a slower pace than expected from the GompertzMakeham law. This mortality deceleration eventually produces "late-life mortality leveling-off" and "late-life mortality plateaus" at extreme old ages (Curtsinger et al., 1992; Economos, 1979; 1983; Gavrilov and Gavrilova, 1991; Greenwood and Irwin, 1939; Vaupel et al., 1998). Actuaries (including Gompertz himself) first noted this phenomenon and proposed a logistic formula for mortality growth with age in order to account for mortality fall-off at advanced ages (Perks, 1932; Beard, 1959; 1971). Greenwood and Irwin (1939) provided a detailed description of this phenomenon in humans and even made the first estimates for the asymptotic value of human mortality (see also review by Olshansky, 1998). According to their estimates, the mortality kinetics of long-lived individuals is close to the law of radioactive decay with half-time approximately equal to 1 year.

The same phenomenon of "almost nonaging" survival dynamics at extreme old ages is detected in many other biological species. In some species the mortality plateau can occupy a sizable part of their life (see Figure 5.5).

Biologists have been well aware of mortality leveling-off since the 1960s. For example, Lindop (1961) and Sacher (1966) discussed mortality deceleration in mice. Strehler and Mildvan (1960) considered mortality deceleration at advanced ages as a prerequisite for all mathematical models of aging to explain. Later A. Economos published a series of articles claiming a priority in the discovery of a "non-Gompertzian paradigm of mortality" (Economos, 1979; 1980; 1983; 1985). He found that mortality leveling-off is observed in rodents (guinea pigs, rats, mice) and invertebrates (nematodes, shrimps, bdelloid rotifers, fruit flies, degenerate medusae Campanularia Flexuosa). In the 1990s the phenomenon of mortality deceleration and leveling-off became widely known after some publications demonstrated mortality leveling-off in large samples of Drosophila melanogaster (Curtsinger et al., 1992) and medflies Ceratitis capitata (Carey et al., 1992), including isogenic strains of Drosophila (Curtsinger et al., 1992; Fukui et al., 1993; 1996). Mortality plateaus at advanced ages are observed for some other insects: house fly Musca vicina and blowfly Calliphora erythrocephala (Gavrilov, 1980), bruchid 


\section{Models of Systems Failure in Aging}

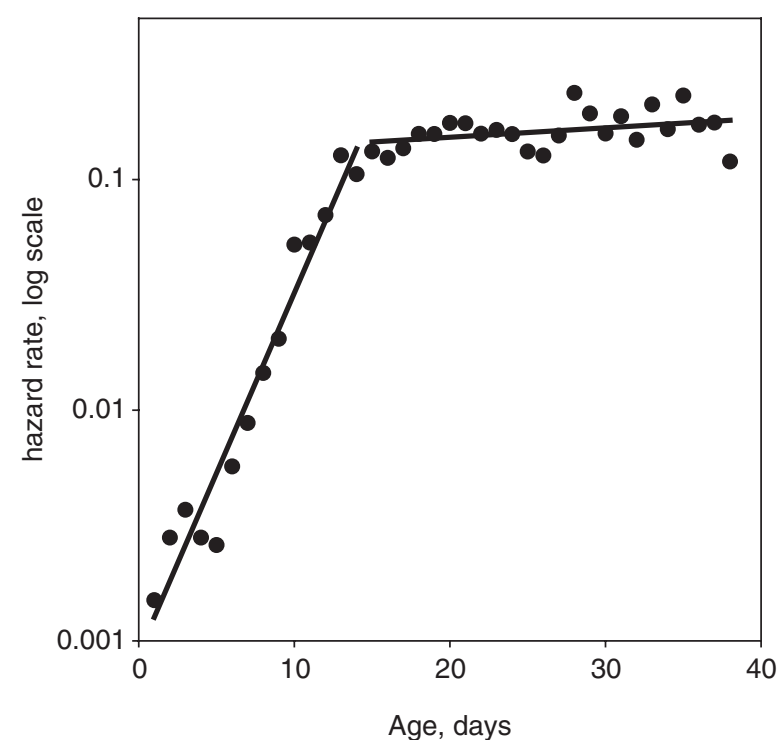

Figure 5.5. Mortality leveling-off in a population of 4,650 male house flies, Hazard rates were computed using life table of house fly, Musca domestica, published by Rockstein and Lieberman (1959). Source: Gavrilov and Gavrilova, 2005.

beetle Callosobruchus maculates (Tatar et al., 1993), fruit flies Anastrepha ludens, Anastrepha obliqua, Anastrepha serpentine and a parasitoid wasp Diachasmimorpha longiacaudtis (Vaupel et al., 1998).

Interestingly, the failure kinetics of manufactured products (steel samples, industrial relays, and motor heat insulators) also demonstrates the same "nonaging" pattern at the end of their "lifespan" (Economos, 1979). This phenomenon is presenting a theoretical challenge to many models and theories of aging. One interesting corollary from these intriguing observations is that there seems to be no fixed upper limit for individual lifespan (Gavrilov, 1984; Gavrilov and Gavrilova, 1991; Wilmoth, 1997).

This observation calls for a very general explanation of this apparently paradoxical "no aging at extreme ages" phenomenon, which will be discussed in this chapter.

Another empirical observation, the compensation law of mortality, in its strong form refers to mortality convergence, when higher values for the parameter $\alpha$ (in the Gompertz function) are compensated by lower values of the parameter $R$ in different populations of a given species:

$$
\ln (R)=\ln (M)-B \alpha
$$

where $B$ and $M$ are universal species-specific invariants. Sometimes this relationship is also called the StrehlerMildvan correlation (Strehler, 1978; Strehler and Mildvan, 1960), although that particular correlation was largely an artifact of the opposite biases in parameters estimation caused by not taking into account the age-independent mortality component, the Makeham

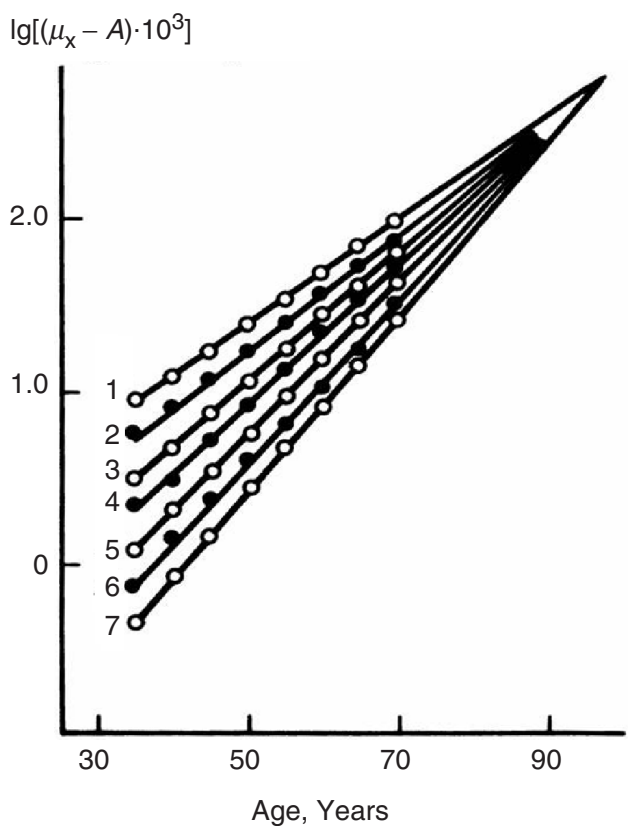

Figure 5.6. Compensation Law of Mortality. Convergence of mortality rates in different populations at advanced ages. Death rates (with removed age-independent external mortality component) are plotted in a log scale as a function of age in the following countries: 1 - India, 1941-1950, males. 2 - Turkey, 1950-1951, males. 3 - Kenya, 1969, males. 4 - Northern Ireland, 1950-1952, males. 5 - England and Wales, 1930-1932, females. 6 - Austria, 1959-1961, females. 7 - Norway, 1956-1960, females. Adapted from Gavrilov and Gavrilova, "The Biology of Life Span," 1991.

term $A$ (see Gavrilov and Gavrilova, 1991; Golubev, 2004). Parameter $B$ is called the species-specific lifespan (95 years for humans), and parameter $M$ is called the species-specific mortality rate $\left(0.5\right.$ year $^{-1}$ for humans). These parameters are the coordinates for convergence of all the mortality trajectories into one single point (within a given biological species), when extrapolated by the Gompertz function (Gavrilov and Gavrilova, 1979; 1991). This means that high mortality rates in disadvantaged populations (within a given species) are compensated for by a low apparent "aging rate" (longer mortality doubling period). As a result of this compensation, the relative differences in mortality rates tend to decrease with age within a given biological species (Figure 5.6).

In those cases when the compensation law of mortality is not observed in its strong form, it may still be valid in its weak form-i.e., the relative differences in mortality rates of compared populations tend to decrease with age in many species. Explanation of the compensation law of mortality is a great challenge for many theories of aging and longevity (Gavrilov and Gavrilova, 1991; Strehler, 1978).

There are some exceptions from both the Gompertz law of mortality and the compensation law of mortality 
that have to be understood and explained. There were reports that in some cases the organisms die according to the Weibull (power) law (see the section on basic failure models). The Weibull law is more commonly applicable to technical devices (Barlow and Proschan, 1975; Rigdon and Basu, 2000; Weibull, 1951), while the Gompertz law is more common in biological systems (Finch, 1990; Gavrilov and Gavrilova, 1991; Strehler, 1978). As was already noted, the exponential Gompertzian increase in age-specific mortality is observed for many biological species including fruit flies Drosophila melanogaster, nematodes, mosquitoes, human lice, flour beetles, mice, rats, dogs, horses, mountain sheep, baboons and humans. Comparative meta-analysis of 129 life tables for fruit flies as well as 285 life tables for humans demonstrates that the Gompertz law of mortality provides a much better data fit for each of these two biological species, compared to the Weibull law (Gavrilov and Gavrilova, 1991, pp. 55-56, 68-72). Possible explanations why organisms prefer to die according to the Gompertz law, while technical devices typically fail according to the Weibull law are provided elsewhere (Gavrilov and Gavrilova, 1991; 2001; 2005) and will be discussed later in this chapter (see the section on theoretical models of systems failure in aging).

Both the Gompertz and the Weibull failure laws have a fundamental explanation rooted in reliability theory (Barlow and Proschan, 1975) and are the only two theoretically possible limiting extreme value distributions for systems whose lifespans are determined by the first failed component (Gumbel, 1958; Galambos, 1978). In other words, as the system becomes more and more complex (contains more vital components, each being critical for survival), its lifespan distribution may asymptotically approach one of the only two theoretically possible limiting distributions - either Gompertz or Weibull (depending on the early kinetics of failure of system components). The two limit theorems in the statistics of extremes (Gumbel, 1958; Galambos, 1978) make the Gompertz and the Weibull failure laws as fundamental as are some other famous limiting distributions known in regular statistics, e.g., the normal distribution and the Poisson distribution. It is puzzling, however, why organisms prefer to die according to the Gompertz law, while technical devices typically fail according to the Weibull law. One possible explanation of this mystery is suggested later in this chapter.

Thus, a comprehensive theory of species aging and longevity should provide answers to the following questions:

1. Why do most biological species deteriorate with age (i.e., die more often as they grow older) while some primitive organisms do not demonstrate such a clear mortality growth with age (Austad, 2001; Finch, 1990; Haranghy and Balázs, 1980; Martinez, 1998)?
2. Specifically, why do mortality rates increase exponentially with age in many adult species (Gompertz law)? How should we handle cases when the Gompertzian mortality law is not applicable?

3. Why does the age-related increase in mortality rates vanish at older ages? Why do mortality rates eventually decelerate compared to predictions of the Gompertz law, occasionally demonstrate leveling-off (late-life mortality plateau), or even a paradoxical decrease at extreme ages?

4. How do we explain the so-called compensation law of mortality (Gavrilov and Gavrilova, 1991)?

Any theory of human aging has to explain these last three rules, known collectively as mortality, or failure, laws. And reliability theory, by way of a clutch of equations, covers all of them (see the section on theoretical models of systems failure in aging, and Gavrilov and Gavrilova, 1991, 2001, 2005).

\section{DECLINE IN SYSTEMS' REDUNDANCY WITH AGE}

Many age changes in living organisms can be explained by cumulative effects of cell loss over time. For example, the very common phenomenon of hair graying with age is caused by depletion of hair follicle melanocytes (Commo et al., 2004). Melanocyte density in human epidermis declines gradually with age at a rate approximately $0.8 \%$ per year (Gilchrest et al., 1979). Hair graying is a relatively benign phenomenon, but cell loss can also lead to more serious consequences.

Recent studies found that such conditions as atherosclerosis, atherosclerotic inflammation, and consequent thromboembolic complications could be linked to agerelated exhaustion of progenitor cells responsible for arterial repair (Goldschmidt-Clermont, 2003; Libby, 2003; Rauscher et al., 2003). Taking these progenitor cells from young mice and adding them to experimental animals prevents atherosclerosis progression and atherosclerotic inflammation (Goldschmidt-Clermont, 2003; Rauscher et al., 2003).

Age-dependent decline in cardiac function is also linked to the failure of cardiac stem cells to replace dying myocytes with new functioning cells (Capogrossi, 2004). It was found that aging-impaired cardiac angiogenic function could be restored by adding endothelial precursor cells derived from the young bone marrow (Edelberg et al., 2002).

Chronic renal failure is known to be associated with decreased number of endothelial progenitor cells (Choi et al., 2004). People with diminished numbers of nephrons in their kidneys are more likely to suffer from hypertension (Keller et al., 2003), and the number of glomeruli decreases with human age (Nyengaard and Bendtsen, 1992).

Humans generally lose $30-40 \%$ of their skeletal muscle fibers by age 80 (Leeuwenburgh, 2003), which 


\section{Models of Systems Failure in Aging}

contributes to such adverse health outcomes as sarcopenia and frailty. Loss of striated muscle cells in such places as rhabdosphincter from $87.6 \%$ in a 5-week-old child to only $34.2 \%$ in a 91 -year-old has obvious implications for urological failure-incontinence (Strasser, 2000).

A progressive loss of dopaminergic neurons in substantia nigra results in Parkinson's disease, loss of GABAergic neurons in striatum produces Huntington's disease, loss of motor neurons is responsible for amyotrophic lateral sclerosis, and loss of neurons in cortex causes Alzheimer's disease over time (Baizabal et al., 2003). A study of cerebella from normal males aged 19-84 years revealed that the global white matter was reduced by $26 \%$ with age, and a selective $40 \%$ loss of both Purkinje and granule cells was observed in the anterior lobe. Furthermore a $30 \%$ loss of volume, mostly due to a cortical volume loss, was found in the anterior lobe, which is predominantly involved in motor control (Andersen et al., 2003).

The phenomenon of human aging of menopause also is caused by loss of ovarian cells. For example, the female human fetus at age 4-5 months possesses 6-7 million eggs (oocytes). By birth, this number drops to 1-2 million and declines even further. At the start of puberty in normal girls, there are only $0.3-0.5$ million eggs - just only $4-8 \%$ of initial numbers (Gosden, 1985; Finch and Kirkwood, 2000; Wallace and Kelsey, 2004). It is now well established that the exhaustion of the ovarian follicle numbers over time is responsible for menopause (reproductive aging and failure), and women having higher ovarian reserve have longer reproductive lifespans (Wallace and Kelsey, 2004). When young ovaries were transplanted to old post-reproductive mice, their reproductive function was restored for a while (Cargill et al., 2003). This example illustrates a general idea that aging largely occurs because of cell loss, which starts early in life.

Loss of cells with age is not limited to the human species and is observed in other animals as well. For example, a nematode $C$. elegans demonstrates a gradual, progressive deterioration of muscle, resembling human sarcopenia (Herndon et al., 2002). The authors of this study also found that the behavioral ability of nematodes was a better predictor of life expectancy than chronological age.

Interestingly, caloric restriction can prevent cell loss (Cohen et al., 2004; McKiernan et al., 2004), which may explain why caloric restriction delays the onset of numerous age-associated diseases and can significantly increase lifespan in mammals (Masoro, 2003).

In terms of reliability theory the loss of cells with age is a loss of system redundancy, and therefore this chapter will focus further on the effects of redundancy loss on systems aging and failure.

\section{Causes of Failure Rate Increase with Age}

\section{THE ORIGIN OF AGE-RELATED INCREASE IN FAILURE RATES}

The aging period for most species occupies the greater part of their lifespan; therefore any model of mortality must explain the existence of this period. It turns out that the phenomena of mortality increase with age and the subsequent mortality leveling-off are theoretically predicted to be an inevitable feature of all reliability models that consider aging as a progressive accumulation of random damage (Gavrilov and Gavrilova, 1991). The detailed mathematical proof of this prediction for some particular models is provided elsewhere (Gavrilov and Gavrilova, 1991; 2001) and is briefly described in the next sections of this chapter.

The simplest schema, which demonstrates an emergence of aging in a redundant system, is presented in Figure 5.7.

If the destruction of an organism occurs not in one but in two or more sequential random stages, this is sufficient for the phenomenon of aging (mortality increase) to appear and then to vanish at older ages. Each stage of destruction corresponds to one of the organism's vitally important structures being damaged. In the simplest organisms with unique critical structures, this damage usually leads to death. Therefore, defects in such organisms do not accumulate, and the organisms themselves do not age - they just die when damaged. For example, the inactivation of microbial cells and spores exposed to a hostile environment (such as heat) follows approximately a nonaging mortality kinetics; their semilogarithmic survival curves are almost linear (Peleg et al., 2003). This observation of nonaging survival
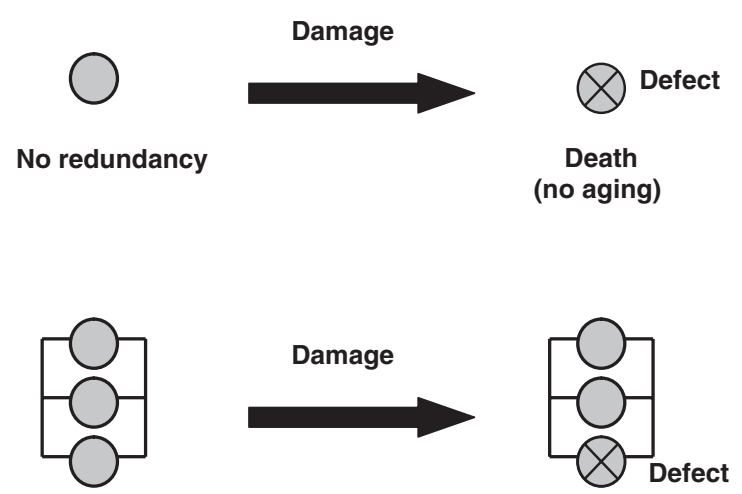

Redundancy

Damage accumulation (aging)

Figure 5.7. Redundancy creates both damage tolerance and damage accumulation (aging). Systems without redundancy (on the top) fail every time when they are damaged, and therefore damage is not accumulated among survivors (no aging). Redundant systems (on the bottom) can sustain damage because of their redundancy, but this damage tolerance leads to damage accumulation (aging). 
dynamics is extensively used in the calculation of the efficacy of sterilization processes in medicine and food preservation (Brock et al., 1994; Davis et al., 1990; Jay, 1996). A similar nonaging pattern of inactivation kinetics is often observed for viruses (Andreadis and Palsson, 1997; Kundi, 1999) and enzymes (Kurganov, 2002; Gouda et al., 2003).

In more complex systems with many vital structures and significant redundancy, every occurrence of damage does not lead to death (unless the environment is particularly hostile) because of their redundancy. Defects accumulate, therefore, giving rise to the phenomenon of aging (mortality increase). Thus, aging is a direct consequence (trade-off) of a system's redundancies, which ensure increased reliability and an increased lifespan of more complex organisms. As defects accumulate, the redundancy in the number of elements finally disappears. As a result of this redundancy exhaustion, the organism degenerates into a system with no redundancy (that is, a system with elements connected in series, in which any new defect leads to death). In such a state, no further accumulation of damage can be achieved, and the mortality rate levels off.

Reliability theory predicts that a system may deteriorate with age even if it is built from nonaging elements with a constant failure rate. The key issue here is the system's redundancy for irreplaceable elements, which is responsible for the aging phenomenon. In other words, each particular step of system destruction/deterioration may seem to be apparently random (no aging, just occasional failure by chance), but if a system failure requires a sequence of several such steps (not just a single step of destruction), then the system as a whole may have an aging behavior.

The positive effect of systems' redundancy is damage tolerance, which decreases the risk of failure (mortality) and increases lifespan. However damage tolerance makes it possible for damage to be tolerated and accumulated over time, thus producing the aging phenomenon.

The next section provides a mathematical illustration for these ideas.

\section{THE SIMPLEST RELIABILITY MODEL OF AGING}

In this section we show that a system built of nonaging components demonstrates an aging behavior (mortality growth with age) and subsequent mortality leveling-off.

Consider a parallel system built of $n$ nonaging elements with a constant failure rate $\mu$ and reliability (survival) function $e^{-\mu x}$ (see also Figure 5.3b). We already showed (see the system's failure and reliability structure section) that in this case the reliability function of the entire parallel system is

$$
S(x)=1-(1-p)^{n}=1-\left(1-e^{-\mu x}\right)^{n}
$$

This formula corresponds to the simplest case when the failure of elements is statistically independent. More complex models would require specific assumptions or prior knowledge on the exact type of interdependence in elements failure. One such model known as "the model of the avalanche-like destruction" is described elsewhere (see pp. 246-251 in Gavrilov, Gavrilova, 1991) and is briefly summarized in the theoretical models of systems failure in aging section.

Consequently, the failure rate of the entire system $\mu_{\mathrm{s}}(x)$, can be written as follows:

$$
\begin{aligned}
\mu_{\mathrm{s}}(x) & =\frac{-d S(x)}{S(x) d x}=\frac{n \mu e^{-\mu x}\left(1-e^{-\mu x}\right)^{n-1}}{1-\left(1-e^{-\mu x}\right)^{n}} \\
& \approx n \mu^{n} x^{n-1}
\end{aligned}
$$

when $x \ll 1 / \mu$ (early-life period approximation, when $\left.1-e^{-\mu x} \approx \mu x\right)$

$$
\approx \mu
$$

when $x \gg 1 / \mu$ (late-life period approximation, when $\left.1-e^{-\mu x} \approx 1\right)$.

Thus, the failure rate of a system initially grows as a power function $n$ of age (the Weibull law). Then the tempo at which the failure rate grows declines, and the failure rate approaches asymptotically an upper limit equal to $\mu$.

Here we should pay attention to three significant points. First, a system constructed of nonaging elements is now behaving like an aging object: aging is a direct consequence of the redundancy of the system (redundancy in the number of elements). Second, at very high ages the phenomenon of aging apparently disappears (failure rate levels off), as redundancy in the number of elements vanishes. The failure rate approaches an upper limit, which is totally independent of the initial number of elements, but coincides with the rate of their loss (parameter $\mu$ ). Third, the systems with different initial levels of redundancy (parameter $n$ ) will have very different failure rates in early life, but these differences will eventually vanish as failure rates approach the upper limit determined by the rate of elements' loss (parameter $\mu$ ). Thus, the compensation law of mortality (in its weak form) is an expected outcome of this illustrative model.

Note also that the identical parallel systems in this example do not die simultaneously when their elements fail by chance. A common view in biology is the idea that all the members of homogeneous population in a hypothetical constant environment should die simultaneously so that the survival curve of such a population would look like a rectangle. This idea stems from the basic principles of quantitative genetics, which assume implicitly that every animal of a given genotype has the same genetically determined lifespan so that all variation of survival time around a genotype mean results from the environmental variance. George Sacher (1977) pointed out that this concept is not applicable to longevity and used an analogy with radioactive decay in his arguments. 


\section{Models of Systems Failure in Aging}

Even the simplest parallel system has a specific lifespan distribution determined entirely by a stochastic nature of the aging process. In order to account for this stochasticity it was proposed to use a stochastic variance component of lifespan in addition to genetic and environmental components of phenotypic lifespan variance (Gavrilov and Gavrilova, 1991). The stochastic nature of the system's destruction also produces heterogeneity in an initially homogeneous population. This kind of induced heterogeneity was observed in isogenic strains of nematodes, in which aging resulted in substantial heterogeneity in behavioral capacity among initially homogeneous worms kept in controlled environmental conditions (Herndon et al., 2002).

The graph shown in Figure 5.8 depicts mortality trajectories for five systems with different degrees of redundancy.

System 1 has only one unique element (no redundancy), and it has the highest failure rate, which does not depend on age (no aging). System 2 has two elements connected in parallel (one extra element is redundant), and the failure rate initially increases with age (aging

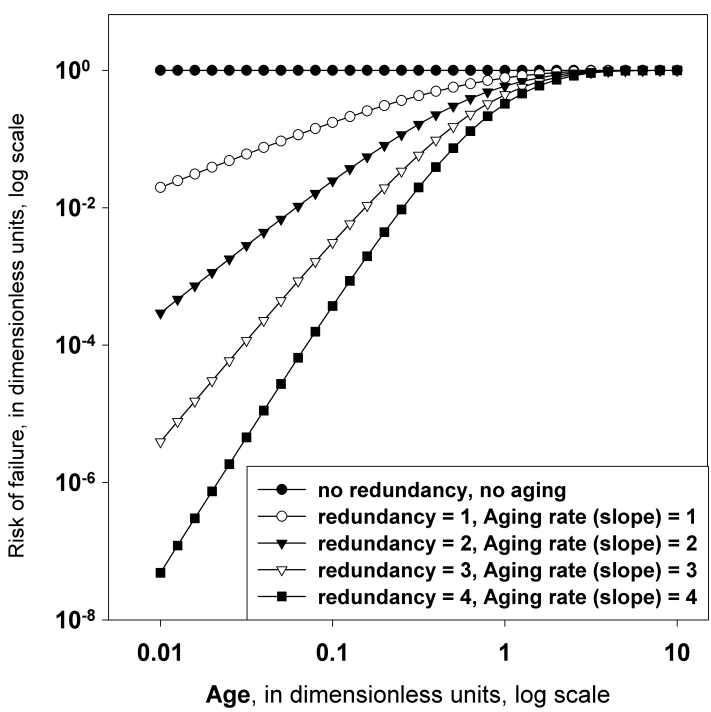

Figure 5.8. Failure kinetics of systems with different levels of redundancy. The dependence of the logarithm of mortality force (failure rate) on the logarithm of age in five systems with different levels of redundancy (computer simulation experiment). The scales for mortality rates (vertical axis) and for age (horizontal axis) are presented in dimensionless units $\left(\mu_{\mathrm{s}} / \mu\right)$ for mortality rates, and $\mu \mathrm{x}$ for age), to ensure the generalizability of the results (invariance of graphs on failure rate of the elements in the system, parameter $\mu$ ). Also, the log scale is used to explore the system behavior in a wide range of ages (0.01-10 units) and failure rates ( $0.00000001-1.0$ units). Dependence 1 is for the system containing only one unique element (no redundancy). Dependence 2 is for the system containing two elements connected in parallel (degree of redundancy $=1$ ). Dependencies 3, 4 and 5 are for systems containing, respectively, 3, 4 and 5 elements connected in parallel (with increasing levels of redundancy). Source: Gavrilov and Gavrilova, 2005. appears). The apparent rate of aging can be characterized by a slope coefficient that is equal to 1 . Finally, the failure rate levels off at advanced ages. Systems 3, 4, and 5 have, respectively, three, four, and five elements connected in parallel (two, three, and four extra elements are redundant), and the failure rate initially increases with age at an apparent aging rate (slope coefficient) of 2, 3, and 4, respectively. Finally, the mortality trajectories of each system level off at advanced ages at exactly the same upper limit to the mortality rate.

This computational example illustrates the following statements: (1) Aging is a direct consequence of a system's redundancy, and the expression of aging is directly related to the degree of a system's redundancy. Specifically, an apparent relative aging rate is equal to the degree of redundancy in parallel systems. (2) All mortality trajectories tend to converge with age, so that the compensation law of mortality is observed. (3) All mortality trajectories level off at advanced ages, and a mortality plateau is observed. Thus, the major aging phenomena (aging itself, the compensation law of mortality, late-life mortality deceleration, and late-life mortality plateaus) are already observed in the simplest redundant systems. However, to explain the Gompertz law of mortality, an additional idea of initial damage load should be taken into account (see next section).

\section{Theoretical Models of Systems Failure in Aging}

\section{HIGHLY REDUNDANT SYSTEM REPLETE WITH DEFECTS}

It was demonstrated in the previous section that the failure rate of a simple parallel system grows with age according to the Weibull law. This model analyzed initially ideal structures in which all the elements are functional from the outset. This standard assumption may be justified for technical devices manufactured from pretested components, but it is not justified for living organisms, replete with initial defects (see Gavrilov and Gavrilova, 1991; 2001; 2004b; 2005).

Following the tradition of the reliability theory, we start our analysis with reliability of an individual system (or homogeneous population). This model of series-parallel structure with distributed redundancy was suggested by Gavrilov and Gavrilova in 1991 and described in more detail in 2001.

Consider first a series-parallel model in which initially functional elements occur very rarely with low probability $q$, so that the distribution of the organism's subsystems (blocks) according to the initial functioning elements they contain is described by the Poisson law with parameter $\lambda=n q$. Parameter $\lambda$ corresponds to the mean number of initially functional elements in a block. 
As has already been noted, the failure rate of a system constructed out of $m$ blocks connected in series is equal to the sum of the failure rates of these blocks, $\mu_{\mathrm{b}}$ (Barlow et al., 1965):

$$
\mu_{\mathrm{s}}=\sum \mu_{\mathrm{b}}=\sum_{i=1}^{n} m P_{i} \mu_{\mathrm{b}}(i)=m C e^{-\lambda} \sum_{i=1}^{n} \frac{\lambda^{i} \mu_{\mathrm{b}}(i)}{i !}
$$

where $P_{i}$ is the probability of a block to have $i$ initially functioning elements. Parameter $C$ is a normalizing factor that ensures the sum of the probabilities of all possible outcomes being equal to unity (see Gavrilov, Gavrilova, 1991; 2001). For sufficiently high values of $n$ and $\lambda$, the normalizing factor turns out to be hardly greater than unity.

Using the formula for failure rate of a block of elements connected in parallel (see the simplest reliability model of aging section), we obtain the final expression for the series-parallel system with distributed redundancy:

$$
\mu_{\mathrm{s}}=\mu \lambda m C e^{-\lambda} \sum_{i=1}^{n} \frac{(\lambda \mu x)^{i-1}}{(i-1) !} \approx R\left(e^{\alpha x}-\varepsilon(x)\right) \approx R e^{\alpha x}
$$

where $R=C m \lambda \mu e^{-\lambda}, \alpha=\lambda \mu$

$\varepsilon(x)$ is close to zero for large $n$ and small $x$ (initial period of life; see Gavrilov, Gavrilova, 1991, 2001 for more detail).

In the early-life period (when $x \ll 1 / \mu$ ) the mortality kinetics of this system follows the exponential Gompertzian law.

In the late-life period (when $x \gg 1 / \mu$ ), the failure rate levels off and the mortality plateau is observed:

$$
\mu_{\mathrm{s}}(x) \approx m \mu
$$

If the age-independent mortality $(A)$ also exists in addition to the Gompertz function, we obtain the wellknown Gompertz-Makeham law described earlier. At advanced ages the rate of mortality decelerates and approaches asymptotically an upper limit equal to $m \mu$.

The model explains not only the exponential increase in mortality rate with age and the subsequent leveling-off, but also the compensation law of mortality:

$$
\ln (R)=\ln (C m \alpha)-\frac{\alpha}{\mu}=\ln (M)-B \alpha
$$

where $M=C m \alpha, B=1 / \mu$.

According to this model, the compensation law is inevitable whenever differences in mortality arise from differences in the parameter $\lambda$ (the mean number of initially functional elements in the block), while the "true aging rate" (rate of elements' loss, $\mu$ ) is similar in different populations of a given species (presumably because of homeostasis). In this case, the species-specific lifespan estimated from the compensation law as an expected age at mortality convergence (95 years for humans, see Gavrilov and Gavrilova, 1991) characterizes the mean lifetime of the elements $(1 / \mu)$.
The model also predicts certain deviations from the exact mortality convergence in a specific direction because the parameter $M$ proved to be a function of the parameter $\alpha$ according to this model (see earlier). This prediction could be tested in future studies.

It also follows from this model that even small progress in optimizing the processes of ontogenesis and increasing the numbers of initially functional elements $(\lambda)$ can potentially result in a remarkable fall in mortality and a significant improvement in lifespan.

The model assumes that most of the elements in the system are initially nonfunctional. This interpretation of the assumption can be relaxed, however, because most nonfunctional elements (e.g., cells) may have already died and been eliminated by the time the adult organism is formed. In fact, the model is based on the hypothesis that the number of functional elements in the blocks is described by the Poisson distribution, and the fate of defective elements and their death in no way affects the conclusions of the model. Therefore, the model may be reformulated in such a way that stochastic events in early development determine later-life aging and survival through variation in initial redundancy of organs and tissues (see, for example, Finch and Kirkwood, 2000). Note that this model does not require an assumption of initial population heterogeneity in failure risks. Instead the model is focused on distributed redundancy of physiological systems within a given organism, or a group of initially identical organisms.

\section{PARTIALLY DAMAGED REDUNDANT SYSTEM}

In the preceding section, we examined a reliability model for a system consisting of $m$ series-connected blocks with numbers of elements distributed according to the Poisson law. In this section, we consider a more general case in which the probability of an element being initially functional can take any possible value: $0<q \leq 1$ (see Gavrilov and Gavrilova, 1991; 2001 for more detail).

In the general case, the distribution of blocks in the organism according to the number of initially functional elements is described by the binomial rather than Poisson distribution.

If an organism can be presented as a system constructed of $m$ series-connected blocks with binomially distributed elements, its failure rate is given by the following formula:

$$
\begin{aligned}
\mu_{\mathrm{s}} \approx & \operatorname{Cmn}(q \mu)^{n}\left[\frac{1-q}{q \mu}+x\right]^{n-1}=\operatorname{Cmn}(q \mu)^{n}\left(x_{0}+x\right)^{n-1} \\
& \text { where } x_{0}=\frac{1-q}{q \mu}
\end{aligned}
$$

It is proposed to call a parameter $x_{0}$ the initial virtual age of the system, IVAS (Gavrilov and Gavrilova, 1991; 2001). Indeed, this parameter has the dimension of time, and corresponds to the age by which an initially ideal system would have accumulated as many defects as a real 


\section{Models of Systems Failure in Aging}

system already has at the initial moment in time (at $x=$ $0)$. In particular, when $q=1$, i.e., when all the elements are functional at the beginning, the initial virtual age of the system is zero and the failure rate grows as a power function of age (the Weibull law), as described in the causes of failure rate increase with age section. However, when the system is not initially ideal $(q<1)$, we obtain the binomial law of mortality (see basic failure models).

In the case when $x_{0}>0$, there is always an initial period of time, such that $x \ll x_{0}$ and the following approximation to the binomial law is valid:

$$
\begin{aligned}
\mu_{\mathrm{s}} & \approx C m n(q \mu)^{n} x_{0}^{n-1}\left[1+\frac{x}{x_{0}}\right]^{n-1} \\
& \approx C m n(q \mu)^{n} x_{0}^{n-1} \exp \left[\frac{n-1}{x_{0}} x\right]
\end{aligned}
$$

Hence, for any value of $q<1$ there always exists a period of time $x$ when the number of newly formed defects is much less than the original number, and the failure rate grows exponentially with age.

So, if the system is not initially ideal $(q<1)$, the failure rate in the initial period of time grows exponentially with age according to the Gompertz law. A numerical example provided in Figure 5.2 (see the reliability approach to system's failure in aging section) shows that increase in the initial system's damage load (initial virtual age) converts the observed mortality trajectory from the Weibull to the Gompertz one.

The model discussed here not only provides an explanation for the exponential increase in the failure rate with age, but it also explains the compensation law of mortality (see Gavrilov and Gavrilova, 1991; 2001).

The compensation law of mortality is observed whenever differences in mortality are caused by differences in initial redundancy (the number of elements in a block, $n$ ), while the other parameters, including the "true aging rate" (rate of elements' loss $\mu$ ), are similar in populations of a given species (presumably because of homeostasis - stable body temperature, glucose concentration, etc.). For lower organisms with poor homeostasis there may be deviations from this law. Our analysis of data published by Pletcher et al. (2000) revealed that in Drosophila this law holds true for male-female comparisons (keeping temperature the same), but not for experiments conducted at different temperatures, presumably because temperature may influence the rate of element loss.

The failure rate of the blocks asymptotically approaches an upper limit which is independent of the number of initially functional elements and is equal to $\mu$. Therefore the failure rate of a system consisting of $m$ blocks in series tends asymptotically with increased age to an upper limit $m \mu$, independently of the values of $n$ and $q$.
Thus the reliability model described here provides an explanation for a general pattern of aging and mortality in biological species: the exponential growth of failure rate in the initial period, with the subsequent mortality deceleration and leveling-off, as well as the compensation law of mortality.

This model might also be called the model of seriesconnected blocks with varying degrees of redundancy or distributed redundancy. The basic conclusion of the model might be reformulated as follows: if vital components of a system differ in their degree of redundancy, the mortality rate initially grows exponentially with age (according to the Gompertz law) with subsequent levelingoff in later life.

\section{HETEROGENEOUS POPULATION OF REDUNDANT ORGANISMS}

In the previous sections, we examined a situation in which series-connected blocks have varying degrees of redundancy within each organism, while the organisms themselves were considered to be initially identical to each other and to have the same risk of death. This latter assumption can be justified in some special cases (see Gavrilov and Gavrilova, 1991) and also when the focus is on the analysis of the individual risks of failure. In a more general case the population heterogeneity needs to be taken into account, because there is a large variation in the numbers of cells for the organisms of the same species (Finch and Kirkwood, 2000).

In this section, we demonstrate that taking into account the heterogeneity of the population provides an explanation for all the basic laws of mortality. This model of heterogeneous redundant systems was proposed by Gavrilov and Gavrilova in 1991 (pp. 264-272).

The model considers the simplest case when the organism consists of a single vital block with $n$ elements connected in parallel with $q$ being the probability that an element is initially functional. Then the probability of encountering an organism with $i$ initially functional elements out of a total number $n$ of elements is given by the binomial distribution law.

The final formula for failure rate in heterogeneous population, $\mu_{\mathrm{p}}(x)$, is (see Gavrilov and Gavrilova, 1991 for more details):

$$
\begin{aligned}
\mu_{\mathrm{p}}(x) & =\frac{F^{\prime}(x)}{1-F(x)}=\frac{n q \mu e^{-\mu x}\left(1-q e^{-\mu x}\right)^{n-1}}{1-\left(1-q e^{-\mu x}\right)^{n}} \\
& \approx C n q \mu(1-q+q \mu x)^{n-1} \text { for } x \ll 1 / \mu \\
& \approx \mu \text { for } x \gg 1 / \mu
\end{aligned}
$$

where $C$ is a normalizing factor.

Thus the hazard rate of a heterogeneous population at first grows with age according to the binomial law 
of mortality, then asymptotically approaches an upper limit $\mu$ :

$$
\begin{aligned}
\mu_{\mathrm{p}}(x) & \approx C n(q \mu)^{n}\left[\frac{1-q}{q \mu}+x\right]^{n-1} \\
& =\operatorname{Cn}(q \mu)^{n}\left(x_{0}+x\right)^{n-1} \text { for } \mathrm{x} \ll \frac{1}{\mu} \\
\mu_{\mathrm{p}}(x) & \approx \mu \text { for } x \gg \frac{1}{\mu}
\end{aligned}
$$

where $x_{0}=(1-q) / q \mu$, a parameter which we propose to call the initial virtual age of the population. This parameter has the dimension of time, and corresponds to the age by which an initially homogeneous population would have accumulated as many damaged organisms as a real population actually possesses at the initial moment in time (at $x=0$ ). In particular, when $q=1$, i.e., when all the elements in each organism are functional at the outset, the initial virtual age of the population is zero and the hazard rate of population grows as a power function of age (the Weibull law), this being the case described in causes of failure rate increase with age. However when the population is not initially homogeneous $(q<1)$, we arrive at the already mentioned binomial law of mortality. Thus, the heterogeneous population model proposed here can also provide a theoretical justification for the binomial law of mortality.

If a population is initially heterogeneous $(q<1)$, the hazard rate in the initial period of time grows exponentially with age (according to the Gompertz law).

The heterogeneous population model not only provides an explanation for the exponential growth in the failure rate with age, but also the compensation law of mortality (Gavrilov and Gavrilova, 1991). The compensation effect of mortality is observed whenever differences in mortality are brought about by interpopulation differences in the number of elements in the organism $(n)$, while the other parameters, including the rate of aging (the rate of irreversible elements failure $\mu$ ), are similar for all compared populations of a particular species (presumably because of homeostasis in physiological parameters). It is not difficult to see the similarity between this explanation for the compensation effect of mortality and the explanations which emerge from the models of individual system described in preceding sections.

Figure 5.9 presents the age kinetics of failure rate in heterogeneous population where redundancy is distributed by the Poisson law (a special case of binomial distribution) with different mean number of functional elements $(\lambda=1,5,10,15$ and 20$)$.

Note that the logarithm of the failure rate is increasing with age in almost a linear fashion, indicating a reasonable applicability of the Gompertz law in this case. Also note that the slope of the lines is increasing with higher mean redundancy levels $(\lambda)$, and the lines

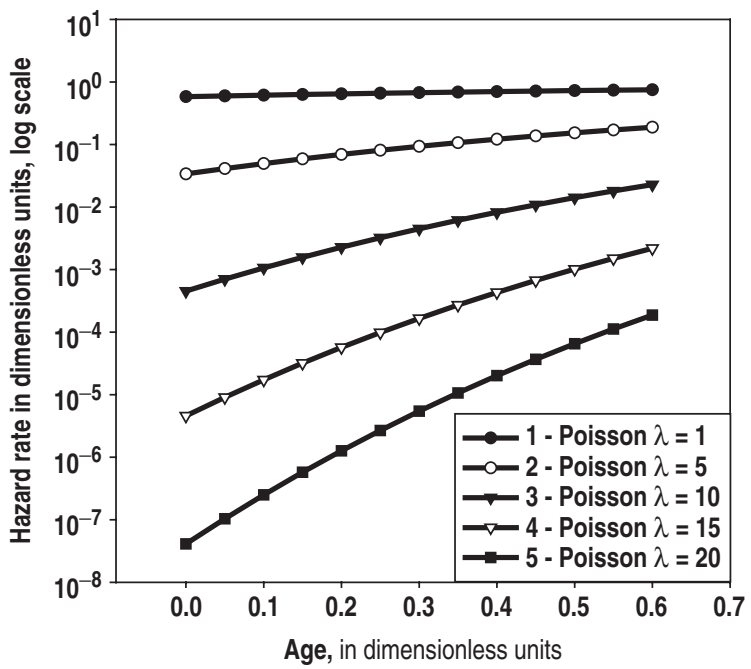

Figure 5.9. Failure kinetics in mixtures of systems with different redundancy levels. Initial period. The dependence of failure rate as a function of age in mixtures of parallel redundant systems having Poisson distribution by initial numbers of functional elements (mean number of elements, $\lambda=1,5,10,15,20$ ). Source: Gavrilov and Gavrilova, 2005

have a tendency for convergence (compensation law of mortality).

The heterogeneous population model leads in principle to the same conclusions as the previously discussed model of series-connected blocks with varying degrees of redundancy. However, we are dealing with two fundamentally different models: whereas in the first model the individual risk of death is the same for all organisms and grows exponentially with age, in the second model there initially exist $n$ subpopulations of living organisms with different risks of death which grow as a power function rather than exponential function of age. However, these different models seem to lead to virtually coincident interpretations of certain mortality phenomena. For example, the compensation effect of mortality is only possible, according to any of the models, when the rate of irreversible age changes is approximately constant within a given species. This interpretation of the compensation effect of mortality is not only a feature of the three models examined in this chapter, but also of other models (Gavrilov, 1978; Gavrilov et al., 1978; Strehler and Mildvan, 1960).

Thus, the heterogeneous population model provides an explanation for all the basic mortality phenomena (the exponential growth of the force of mortality in the initial period, with the subsequent mortality deceleration, as well as the compensation effect of mortality) even in the simplest case when the organism consists of a single vital block with $n$ parallel elements. Generalizing the model to the case of $m$ blocks connected in series in each organism does not present any problems if the blocks are 


\section{Models of Systems Failure in Aging}

independent of each other with respect to their reliability (Gavrilov and Gavrilova, 1991).

\section{MODELS OF AVALANCHE-LIKE DESTRUCTION}

For want of a nail the shoe was lost,

For want of a shoe the horse was lost,

For want of a horse the rider was lost,

For want of a rider the battle was lost,

For want of a battle the kingdom was lost,

And all for the want of a horseshoe nail.

— English nursery rhyme (ca. 1390)

The models described in previous sections assumed that the failures of elements in the organism occur independently of each other. This assumption may be acceptable as the first approximation. In real biological systems many aging phenomena may be represented as a "cascade of dependent failures" which occurs when one of the organism's systems randomly fails (Gavrilov, 1978; Gavrilov et al., 1978). The idea that an avalanche-like mechanism is involved in the destruction of an organism during natural aging is worth further consideration. In fact, it is well-known that defects in an organism have a tendency to multiply following an avalanche-like mechanism. For example, if there are $n$ cancer cells in the organism, each of which is capable of division, the rate at which the organism is transformed into a state with $n+1$ cancer cells increases with the growth of the number of cancer cells $(n)$ already accumulated. Infections of the organism follow similar regularities. The positive feedback between the degree and the rate of an organism's destruction also follows from the fact that when parts of the structure fail, the load on the remaining structures increases, accelerating the wearing-out. It seems that aging may be caused by similar cascades of dependent failures developing over long periods in a hidden, preclinical form. Therefore mathematical models of the avalanche-like destruction of the organism are of particular interest.

Consider the simplest model of the avalanche-like destruction of the organism (Gavrilov and Gavrilova, 1991). Let $S_{0}, S_{1}, \ldots, S_{n}$ denote the states of an organism with $0,1,2, \ldots, n$ defects. Let $\lambda_{0}$ be the background rate at which defects accumulate being independent on the stage of destruction, which the organism has reached. Correspondingly, let $\mu_{0}$ be the age-independent mortality (the Makeham term). In the simplest case, both of these quantities arise from random harmful effects of the external environment. In tandem, there is also an induced rate of deterioration (parameter $\lambda$ ) and an induced failure rate (parameter $\mu$ ) which grow as the number of defects increases. At a first approximation, it can be assumed that both the induced rate of deterioration and the induced failure rate are proportional to the number of defects, so that for an organism with $n$ defects the induced rate

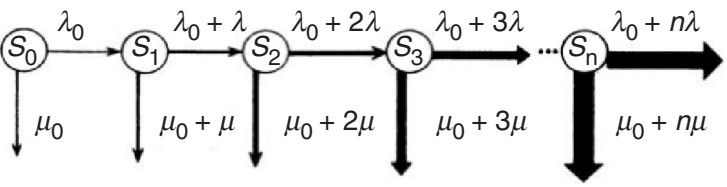

Figure 5.10. Avalanche-like mechanism of organism's destruction with age. In the initial state $\left(S_{0}\right)$ organism has no defects. Then, as a result of random damage, it enters states $S_{1}, S_{2}, \ldots S_{n}$, where $n$ corresponds to the number of defects. Rate of new defects emergence has avalanche-like growth with the number of already accumulated defects (horizontal arrows). Hazard rate (vertical arrows directed down) also has an avalanche-like growth with the number of defects.

of deterioration is equal to $n \lambda$, and the induced failure rate is $n \mu$.

With these assumptions, we can present the avalanchelike destruction of the organism by the schema presented in Figure 5.10.

This schema corresponds to the following system of differential equations:

$$
\begin{aligned}
& d S_{0} / d x=-\left(\lambda_{0}+\mu_{0}\right) \mathrm{S}_{0} \\
& d S_{1} / d x=\lambda_{0} S_{0}-\left(\lambda_{0}+\mu_{0}+\lambda+\mu\right) \mathrm{S}_{1} \\
& d S_{n} / d x=\left[\lambda_{0}+(n-1) \lambda\right] S_{n-1}-\left[\lambda_{0}+\mu_{0}+n(\lambda+\mu)\right] \mathrm{S}_{n}
\end{aligned}
$$

A similar system of equations (not taking into account the age-independent mortality) was obtained and solved in a mathematical model linking the survival of organisms with chromosome damage (Le Bras, 1976). However, this "chromosomal" interpretation of the avalanche model could be applicable only to unicellular organisms, while for multicellular organisms including humans, where chromosomes are compartmentalized in separate cells, this model needs to be revised, or provided with a different "nonchromosomal" interpretation (as it is suggested in this section).

In the particular case when the rate at which defects multiply, the parameter $\lambda$ is significantly greater than the induced failure rate, parameter $\mu,(\lambda \gg \mu)$, the hazard rate of an organism in the initial stage (with low values of $x$ ) grows according to the Gompertz-Makeham law:

$$
\begin{aligned}
& \mu(x) \approx \mu_{0}+\frac{\mu \lambda_{0}\left(1-e^{(\lambda+\mu) x}\right)}{\lambda e^{(\lambda+\mu) x}} \approx A+R e^{\alpha x} \\
& \quad \text { where } A=\mu_{0}-\frac{\mu \lambda_{0}}{\lambda} ; \quad R=\frac{\mu \lambda_{0}}{\lambda} ; \quad \alpha=\lambda+\mu
\end{aligned}
$$

This model of the avalanche-like destruction of the organism not only provides a theoretical justification for the well-known Gompertz-Makeham law, but also explains why the values of the Makeham parameter $A$ sometimes turn out to be negative (when age-independent mortality, $\mu_{0}$, is small as for populations in the developed countries and the background rate of destruction, $\lambda_{0}$, is large).

Another advantage of the avalanche-like destruction model is that it correctly predicts mortality deceleration (deviations from the Gompertz-Makeham law) at very 
old ages. In this extreme age-range, the failure rate grows with age according to the formula

$$
\mu(x) \approx \mu_{0}+\lambda_{0}\left(1-e^{-(\lambda+\mu) x}\right)
$$

Thus the model predicts an asymptotic growth of failure rate with age with an upper limit of $\mu_{0}+\lambda_{0}$.

Alongside the strengths already listed, the avalanchelike destruction model has one significant limitation: it does not conform to the compensation law of mortality in its strong form (Gavrilov and Gavrilova, 1991). Nevertheless, the idea that organisms undergo cascade destruction is one of the promising ideas in further mathematical modeling of aging.

\section{ACCUMULATION OF DEFECTS WITH CONSTANT RATE OF DAMAGE FLOW}

A wide variety of concepts about the destruction of the organism can lead to the model where the rate of damage flow, numerically equal to the mean number of "hits" per unit of time, is practically independent of the state of the organism and is on average constant in time. In the simplest case, the model corresponds to a situation in which the organism is affected by a random flow of traumatic loads with an on average constant rate independent of the state of the organism (exogenous environmental damage like cosmic radiation, viruses, etc.)

However, there is also the possibility of other mechanisms of destruction leading to this particular model of the accumulation of defects. In particular, this model can be obtained after a critical reinterpretation of the assumptions underlying the previously described models. In fact, these models contain an assumption that the death of the organism occurs only when all the elements in a block fail. It is possible that this hypothesis may be justified for some of the organism's systems (stem cell populations, for example). However, in the majority of cases this hypothesis seems contentious. For example, it is hard to imagine that a single surviving liver cell (hepatocyte) can assume the functions of an entire destroyed liver. Significantly more realistic is the hypothesis that the system initially contains an enormous number of elements that greatly exceeds the critical number of defects leading to the death of the organism. In this case we arrive at a schema for the accumulation of damage in which the rate of damage flow (equal to the product of the number of elements and their failure rate) turns out to be practically constant in view of the incommensurability of the high initial numbers of elements, and the much smaller permitted number of defects (Gavrilov and Gavrilova, 1991).

Another advantage of this model is that it allows us to take into account the influence of living conditions on the value for the critical number of defects incompatible with the survival of the organism. The key to the solution of this problem is the replacement of the parallel connection hypothesis (assumed in previously described models) with the more realistic assumption that there exists a critical number of defects incompatible with the survival of the organism. In this case, it is natural to expect that under harsher conditions the critical number of defects leading to death might be less than under more comfortable living conditions. In particular, in the wild, when an animal is deprived of care and forced to acquire its own food, as well as to defend itself against predators, the first serious damage to the organism can lead to death. It is therefore not surprising that the mortality of many animals (in particular, birds) is practically independent of age in the wild. This follows directly from the singlestage destruction of the organism model. On the other hand, the greater the number of defects the organism can accumulate while remaining alive, the greater its lifespan will be.

If the rate of the damage flow equals $k$, and an organism dies after the accumulation of $n$ defects, the density of the survival distribution is identical to the density of the gamma function (see Barlow and Proschan, $1965 ;$ 1975). At the initial moment in time, this distribution corresponds to a power (Weibull) law of mortality with an exponent equal to $(n-1)$.

A fundamentally different result is obtained when the initial damage of organisms is taken into account (Gavrilov and Gavrilova, 1991). If at the initial moment in time the average number of random defects in the population equals $\lambda$, the probability of encountering a living organism, $P_{i}$, with $i$ defects may be approximated by the Poisson law (see Gavrilov and Gavrilova, 1991, pp. 272-276, for more detail).

Since the death of an organism with $i$ defects occurs after $n-i$ additional hits, the density of the lifespan distribution for such organisms is given by

$$
f_{i}(x)=\frac{k(k x)^{n-i-1} e^{-k x}}{(n-i-1) !}, \quad \text { where } \quad i<n
$$

The density of the survival distribution for the whole population, which is a mixture of organisms with $i=0,1$, $2, \ldots n-1$ initial defects, equals

$$
\begin{aligned}
f(x) & =\sum_{i=0}^{n-1} P_{i} f_{i}(x)=C k e^{-(\lambda+k x)} \sum_{i=0}^{n-1} \frac{\lambda^{i}(k x)^{n-i-1}}{i !(n-1-i) !} \\
& =\frac{C k(\lambda+k x)^{n-1} e^{-(\lambda+k x)}}{(n-1) !}
\end{aligned}
$$

It is not difficult to see that at the initial moment in time this model leads to the binomial law of mortality, with an initial virtual age of the population equal to $\lambda /$ $k$. A more detailed analysis of the model is formally similar to the analysis of the other models described in previous sections. We merely note that during the initial time period when $x \ll \lambda / k$, the model leads to an exponential growth of failure rate with age (the Gompertz law) with an exponent, $\alpha$, of $k(n-1) / \lambda$ and a pre-exponential factor, $R$, of $C k \lambda^{n-1} /(n-1)$ !. It is 


\section{Models of Systems Failure in Aging}

easy to see that an inverse relationship between these Gompertz parameters (the compensation effect of mortality) can arise both as a result of variation in parameter $\lambda$ (the degree to which the organisms are initially damaged) and of variation in parameter $n$ (the critical number of defects, dependent on the harshness of living conditions).

Thus the basic mortality phenomena can equally be explained within the framework of the model of accumulation of defects with the constant rate of damage flow, as long as the organisms initially contain a significant number of defects.

Summarizing this brief review of reliability models, we note the striking similarity between the formulas and conclusions of the considered models. It must, however, be noted that we are dealing only with a superficial similarity in behavior between fundamentally different and competing models. The existence of a multitude of competing models is therefore compatible with the reliable and meaningful interpretation of a number of mortality phenomena, since pluralism of models does not preclude their agreement on a number of issues. All these models predict a mortality deceleration, no matter what assumptions are made regarding initial population heterogeneity, or its complete initial homogeneity. Moreover, these reliability models of aging produce mortality plateaus as the inevitable outcome for any values of considered parameters (Gavrilov and Gavrilova, 1991). The only constraint is that the elementary steps of the multistage destruction process of a system should occur only by chance, independent of age. The models also predict that an initially homogeneous population will become highly heterogeneous for risk of death over time (acquired heterogeneity).

\section{Conclusions}

Theoretical reliability models of system failure in aging considered in this book chapter lead to the following conclusions:

1. Redundancy is a key notion for understanding aging and the systemic nature of aging in particular. Systems, which are redundant in numbers of irreplaceable elements, do deteriorate over time (fail more often with age), even if they are built of nonaging elements. The positive effect of systems' redundancy is damage tolerance, which decreases mortality and increases lifespan. However damage tolerance makes it possible for damage to be tolerated and accumulated over time, thus producing the aging phenomenon.

2. An apparent aging rate or expression of aging (measured as age differences in failure rates, including death rates) is higher for systems with higher redundancy levels (all other things being equal). This is an important issue, because it helps to put a correct perspective on fascinating observations of negligible senescence (no apparent aging) observed in the wild and at extreme old ages. Reliability theory explains that some cases of negligible senescence may have a trivial mechanism (lack of redundancies in the system being exposed to challenging environment) and, therefore, will not help to uncover "the secrets of negligible senescence." The studies of negligible senescence make sense, however, when the death rates are also demonstrated to be negligible.

3. Reliability theory also persuades a re-evaluation of the old belief that aging is somehow related to limited economic or evolutionary investments in systems longevity. The theory provides a completely opposite perspective on this issue - aging is a direct consequence of investments into systems reliability and durability through enhanced redundancy. This is a significant statement, because it helps to understand why the expression of aging (differences in failure rates between the younger and the older age groups) may be actually more profound in more complicated redundant systems, designed for higher durability.

4. During the life course the organisms are exhausting the reserve numbers of their cells (Gosden, 1985; Herndon et al., 2002), losing reserve capacity (Bortz, 2002; Sehl and Yates, 2001), and this redundancy depletion explains the observed "compensation law of mortality" (mortality convergence at older ages) as well as the observed late-life mortality deceleration, leveling-off, and mortality plateaus.

5. Living organisms seem to be formed with a high load of initial damage, and therefore their lifespan and aging patterns may be sensitive to early-life conditions that determine this initial damage load during early development. The idea of early-life programming of aging and longevity may have important practical implications for developing early-life interventions promoting health and longevity.

The theory also suggests that aging research should not be limited to the studies of qualitative changes (like age changes in gene expression), because changes in quantity (numbers of cells and other functional elements) could be an important driving force of aging process. In other words, aging may be largely driven by a process of redundancy loss.

The reliability theory predicts that a system may deteriorate with age even if it is built from nonaging elements with constant failure rate. The key issue here is the system's redundancy for irreplaceable elements, which is responsible for the aging phenomenon. In other words, each particular step of system destruction/deterioration may seem to be apparently random (no aging, just occasional failure by chance), but if a system failure 
requires a sequence of several such steps (not just a single step of destruction), then the system as a whole may have an aging behavior.

Why is this important? Because the significance of beneficial health-promoting interventions is often undermined by claims that these interventions are not proven to delay the process of aging itself, but instead simply delay or "cover-up" some particular manifestations of aging.

In contrast to these pessimistic views, reliability theory says that there may be no specific underlying elementary "aging process itself" - instead aging may be largely a property of a redundant system as a whole, because it has a network of destruction pathways, each being associated with particular manifestations of aging (types of failure). Therefore, we should not be discouraged by only partial success of each particular intervention, but instead we can appreciate an idea that we do have so many opportunities to oppose aging in numerous different ways.

Thus, the efforts to understand the routes and the early stages of age-related degenerative diseases should not be discarded as irrelevant to understanding the "true biological aging." On the contrary, the attempts to build an intellectual firewall between biogerontological research and clinical medicine are counterproductive. After all, the main reason why people are really concerned about aging is because it is related to health deterioration and increased morbidity. The most important pathways of age changes are those that make older people sick and frail (Bortz, 2002).

Reliability theory suggests general answers to both the "why" and the "how" questions about aging. It explains "why" aging occurs by identifying the key determinant of aging behavior-system redundancy in numbers of irreplaceable elements. Reliability theory also explains "how" aging occurs, by focusing on the process of redundancy loss over time as the major mechanism of aging.

Ageing is a complex phenomenon (Sehl and Yates, 2001), and a holistic approach using reliability theory may help to analyze, understand, and perhaps to control it. We suggest, therefore, adding theoretical reliability models of system failure in aging to the arsenal of methodological approaches for the studying of human aging.

\section{ACKNOWLEDGMENTS}

This work was supported in part by grants from the National Institute on Aging.

\section{REFERENCES}

Andersen, B.B., Gundersen, H.J., and Pakkenberg B. (2003). Aging of the human cerebellum: A stereological study. J. Comp. Neurol. 466, 356-365.
Andreadis, S., and Palsson, B.O. (1997). Coupled effects of polybrene and calf serum on the efficiency of retroviral transduction and the stability of retroviral vectors. Hum. Gene Ther. 8, 285-291.

Austad, S.N. (2001). Concepts and theories of aging. In Masoro, E.J., and Austad, S.N., Handbook of the Biology of Aging. San Diego, CA: Academic Press, pp. 3-22.

Aven, T., and Jensen, U. (1999). Stochastic Models in Reliability. New York: Springer-Verlag.

Ayyub, B.M., and McCuen, R.H. (2003). Probability, Statistics, Reliability for Engineers and Scientists. Boca Raton, FL: Chapman and Hall/CRC.

Baizabal, J.M., Furlan-Magaril, M., Santa-Olalla, J., and Covarrubias, L. (2003). Neural stem cells in development and regenerative medicine. Arch. Med. Res. 34, 572-588.

Barlow, R.E., and Proschan, F. (1975). Statistical Theory of Reliability and Life Testing. Probability Models. New York: Holt, Rinehart and Winston.

Barlow, R.E., Proschan, F., and Hunter, L.C. (1965). Mathematical Theory of Reliability. New York: John Wiley.

Beard, R.E. (1959). Note on some mathematical mortality models. In: Wolstenholme, G.E.W., and O'Connor, M. (Eds.). The Lifespan of Animals (pp. 302-311). Boston: Little, Brown.

Beard, R.E. (1971). Some aspects of theories of mortality, cause of death analysis, forecasting and stochastic processes. In: Brass, W. (Ed.), Biological Aspects of Demography (pp. 57-68). London: Taylor and Francis.

Bortz, W.M. (2002). A conceptual framework of frailty: A review. J. Gerontol. Ser. A 57, M283-M288.

Brock, T.D., Madigan, M.T., Martinko, J.M., and Parker, J. (1994). Biology of Microorganisms (7th ed.), Englewood Cliffs, NJ: Prentice-Hall.

Bronikowski, A.M., Alberts, S.C., Altmann, J., Packer, C., Carey, K.D., and Tatar, M. (2002). The aging baboon: Comparative demography in a non-human primate. Proc. Natl. Acad. Sci. USA 99, 9591-9595.

Brooks, A., Lithgow, G.J., and Johnson, T.E. (1994). Mortality rates in a genetically heterogeneous population of Caenorhabditis elegans. Science 263, 668-671.

Burns, J., Clarke, G., and Lumsden, C.J. (2002). Photoreceptor death: Spatiotemporal patterns arising from one-hit death kinetics and a diffusible cell death factor. Bull. Math. Biol. 64, 1117-1145.

Calne, D.B. (1994). Is idiopathic parkinsonism the consequence of an event or a process? Neurology 44, 5-10 (1994).

Capogrossi, M.C. (2004). Cardiac stem cells fail with aging: A new mechanism for the age-dependent decline in cardiac function. Circ. Res. 94, 411-413.

Carey, J.R., and Liedo, P. (1995). Sex-specific life table aging rates in large medfly cohorts. Exp. Gerontol. 30, 315-325.

Carey, J.R., Liedo, P., Orozco, D., and Vaupel, J.W. (1992). Slowing of mortality rates at older ages in large Medfly cohorts. Science 258, 457-461.

Cargill, Sh. L., Carey, J.R., Muller, H.-G., and Anderson, G. (2003). Age of ovary determines remaining life expectancy in old ovariectomized mice. Aging Cell 2, 185-190.

Carnes, B.A., Olshansky, S.J., and Grahn, D. (1998). An interspecies prediction of the risk of radiation-induced mortality. Radiat. Res. 149, 487-492.

Choi, J.H., Kim, K.L., Huh, W., Kim, B., Byun, J., Suh, et al. (2004). Decreased number and impaired angiogenic function 


\section{Models of Systems Failure in Aging}

of endothelial progenitor cells in patients with chronic renal failure. Arterioscler. Thromb. Vas.c Biol. 24, 1246-1252.

Clark, A.G., and Guadalupe, R.N. (1995). Probing the evolution of senescence in Drosophila melanogaster with P-element tagging. Genetica 96, 225-234.

Clark, V.A. (1975). Survival distribution. Annual Review of Biophysics and Bioengineering 4, 431-448.

Clarke, G., Collins, R.A., Leavitt, B.R., Andrews, D.F., Hayden, M.R., Lumsden, C.J., and McInnes, R.R. (2000). A one-hit model of cell death in inherited neuronal degenerations. Nature 406, 195-199.

Clarke, G., Collins, R.A., Leavitt, B.R., Andrews, D.F., Hayden, M.R., Lumsden, C.J., and McInnes, R.R. (2001a). Addendum: A one-hit model of cell death in inherited neuronal degenerations. Nature 409, 542.

Clarke, G., Lumsden, C.J., and McInnes, R.R. (2001b). Inherited neurodegenerative diseases: The one-hit model of neurodegeneration. Hum. Mol. Genet. 10, 2269-2275.

Cohen, H.Y., Miller, C., Bitterman, K.J., Wall, N.R., Hekking, B., Kessler, B., et al. (2004). Calorie restriction promotes mammalian cell survival by inducing the SIRT1 deacetylase. Science 305, 390-392.

Commo, S., Gaillard, O., and Bernard, B.A. (2004). Human hair greying is linked to a specific depletion of hair follicle melanocytes affecting both the bulb and the outer root sheath. Br. J. Dermatol. 150, 435-443.

Crowder, M .J., Kimber, A.C., Smith, R.L., and Sweeting, T.J. (1991). Statistical Analysis of Reliability Data. London: Chapman and Hall.

Curtsinger, J.W., Fukui, H., Townsend, D., and Vaupel, J.W. (1992). Demography of genotypes: Failure of the limited life-span paradigm in Drosophila melanogaster. Science 258, 461-463.

Davis, B.D., Dulbeco, R., Eisen, H.N., and Ginsberg, H.S. (1990). Microbiology (4th ed.), Philadelphia, PA: Lippincott.

Eakin, T., Shouman, R., Qi, Y.L., Liu, G.X. and Witten, M. (1995). Estimating parametric survival model parameters in gerontological aging studies. Methodological problems and insights. J. Gerontol. Ser. A 50, B166-B176.

Economos, A.C. (1979). A non-gompertzian paradigm for mortality kinetics of metazoan animals and failure kinetics of manufactured products. AGE 2, 74-76.

Economos, A.C. (1980). Kinetics of metazoan mortality. J. Social Biol. Struct., 3, 317-329.

Economos, A.C. (1983). Rate of aging, rate of dying and the mechanism of mortality. Arch. Gerontol. and Geriatrics $1,3-27$.

Economos, A. (1985). Rate of aging, rate of dying and non-Gompertzian mortality-Encore ... Gerontology 31, 106-111.

Edelberg, J.M., Tang, L., Hattori, K., Lyden, D., and Rafii, S. (2002). Young adult bone marrow-derived endothelial precursor cells restore aging-impaired cardiac angiogenic function. Circ. Res. 90, E89-E93.

Feller, W. (1968). An Introduction to Probability Theory and Its Applications, Vol. 1. New York: Wiley and Sons.

Finch, C.E., (1990). Longevity, senescence and the genome. Chicago: University of Chicago Press.

Finch, C.E., and Kirkwood, T.B. L. (2000). Chance, Development, and Aging. New York, Oxford: Oxford University Press.
Finger, S., Le Vere, T.E., Almli, C.R. and Stein, D.G. (Eds.) (1988). Brain Injury and Recovery: Theoretical and Controversial Issues. New York: Plenum Press.

Fukui, H.H., Ackert, L. and Curtsinger, J.W. (1996). Deceleration of age-specific mortality rates in chromosomal homozygotes and heterozygotes of Drosophila melanogaster. Exp. Gerontol. 31, 517-531.

Fukui, H.H., Xiu, L., and Curtsinger, J.W. (1993). Slowing of age-specific mortality rates in Drosophila melanogaster. Exp. Gerontol. 28, 585-599.

Galambos, J. (1978). The Asymptotic Theory of Extreme Order Statistics. New York: Wiley.

Gavrilov, L.A. (1978). Mathematical model of aging in animals. Doklady Akademii Nauk SSSR Biological Sciences 238, 53-55 (English translation).

Gavrilov, L.A. (1980). Study of Life Span Genetics Using the Kinetic Analysis. Thesis, Moscow, Russia: Moscow State University.

Gavrilov, L.A. (1984). Does a limit of the life span really exist? Biofizika 29, 908-911.

Gavrilov, L.A., and Gavrilova, N.S. (1979). Determination of species length of life. Doklady Akademii Nauk SSSR Biological Sciences 246, 905-908 (English translation).

Gavrilov, L.A., and Gavrilova, N.S. (1991). The Biology of Life Span: A Quantitative Approach. New York: Harwood Academic Publishers.

Gavrilov, L.A., and Gavrilova, N.S. (2001). Biodemographic study of familial determinants of human longevity. Population: English Selection 13, 197-222.

Gavrilov, L.A., and Gavrilova, N.S. (2003a). The quest for a general theory of aging and longevity. Science's SAGE KE (Science of Aging Knowledge Environment), 16 July 2003; 2003(28), 1-10. Available: http://sageke. sciencemag.org

Gavrilov, L.A., and Gavrilova, N.S. (2003b). Early-life factors modulating lifespan. In: Rattan, S.I.S. (Ed.). Modulating Aging and Longevity (pp. 27-50), Dordrecht, The Netherlands: Kluwer Academic Publishers.

Gavrilov, L.A., and Gavrilova, N.S. (2004a). The reliabilityengineering approach to the problem of biological aging. Ann. N.Y. Acad. Sci. 1019, 509-512.

Gavrilov, L.A., and Gavrilova, N.S. (2004b). Early-life programming of aging and longevity: The idea of high initial damage load (the HIDL hypothesis). Ann. N.Y. Acad. Sci. 1019, 496-501.

Gavrilov, L.A., and Gavrilova, N.S. (2004c). Why we fall apart. Engineering's reliability theory explains human aging. IEEE Spectrum 9, 2-7.

Gavrilov, L.A., and Gavrilova, N.S. (2005). Reliability theory of aging and longevity. In: Masoro, E.J., and Austad, S.N., Handbook of the Biology of Aging. San Diego, CA: Academic Press $1-40$.

Gavrilov, L.A., Gavrilova, N.S. and Iaguzhinskii, L.S. (1978). Basic patterns of aging and death in animals from the standpoint of reliability theory. J. General Biology (Zhurnal Obshchej Biologii, Moscow), 39, 734-742 (In Russian).

Gilchrest, B.A., Blog, F.B., and Szabo, G. (1979). Effects of aging and chronic sun exposure on melanocytes in human skin. J. Invest. Dermatol., 73, 141-3.

Goldschmidt-Clermont, P.J. (2003). Loss of bone marrowderived vascular progenitor cells leads to inflammation and atherosclerosis. Am. Heart J. 146(4 Suppl), S5-S12. 
Golubev, A. (2004). Does Makeham make sense? Biogerontology $5,159-167$.

Gompertz, B. (1825). On the nature of the function expressive of the law of human mortality and on a new mode of determining life contingencies. Philos. Trans. Roy. Soc. London A 115, 513-585.

Gosden, R.G. (1985). The Biology of Menopause: The Cause and Consequence of Ovarian Aging. San Diego, CA: Academic Press.

Gouda, M.D., Singh, S.A., Rao, A.G., Thakur, M.S., and Karanth, N.G. (2003). Thermal inactivation of glucose oxidase: Mechanism and stabilization using additives. J. Biol. Chem. 278, 24324-24333.

Greenwood, M., and Irwin, J.O. (1939). The biostatistics of senility. Hum. Biol. 11, 1-23.

Griffiths, A.J.F., Miller, J.H., Suzuki, D.T., Lewontin, R.C., and Gelbart, W.M. (1996). An Introduction to Genetic Analysis (6th ed.). New York: W.H. Freeman.

Gumbel, E.J. (1958). Statistics of Extremes. New York: Columbia University Press.

Hall, J.C. (1969). Age-dependent enzyme changes in Drosophila melanogaster. Exp. Gerontol. 4, 207-222.

Haranghy, L., and Balázs, A. (1980). Regeneration and rejuvenation of invertebrates. In: Shock, N.W. (Ed.), Perspectives in Experimental Gerontology (pp. 224-233). New York: Arno Press.

Heintz, N. (2000). One-hit neuronal death. Nature, 406, 137-138.

Herndon, L.A., Schmeissner, P.J., Dudaronek, J.M., Brown, P.A., Listner, K.M., Sakano, Y., et al. (2002). Stochastic and genetic factors influence tissue-specific decline in ageing C. elegans. Nature 419, 808-814.

Hirsch, H.R., and Peretz, B. (1984). Survival and aging of a small laboratory population of a marine mollusc. Aplysia californica. Mech. Ageing Dev. 27, 43-62.

Hirsch, A.G., Williams, R.J., and Mehl, P. (1994). Kinetics of medfly mortality. Exp. Gerontol. 29, 197-204.

Janse, C., Slob, W., Popelier, C.M., and Vogelaar, J.W. (1988) Survival characteristics of the mollusc Lymnaea stagnalis under constant culture conditions: effects of aging and disease. Mech. Ageing Dev. 42, 263-174.

Jay, J.M. (1996). Modern Food Microbiology. New York: Chapman and Hall.

Johnson, T.E. (1987). Aging can be genetically dissected into component processes using long-lived lines of Caenorhabditis elegans. Proc. Natl. Acad. Sci. USA 84, 3777-3781.

Johnson, T.E. (1990). Increased life span of age-1 mutants in Caenorhabditis elegans and lower Gompertz rate of aging. Science 249, 908-912.

Kaufmann, A., Grouchko, D. and Cruon, R. (1977). Mathematical Models for the Study of the Reliability of Systems. New York: Academic Press.

Keller, G., Zimmer, G., Mall, G., Ritz, E., and Amann, K. (2003). Nephron number in patients with primary hypertension. N. Engl. J. Med. 348, 101-108.

Khazaeli, A.A., Xiu, L., and Curtsinger, J.W. (1995). Stress experiments as a means of investigating age-specific mortality in Drosophila melanogaster. Exp. Gerontol. 30, 177-184.

Khazaeli, A.A., Xiu, L., and Curtsinger, J.W. (1996). Effect of density on age-specific mortality in Drosophila: A density supplementation experiment. Genetica 98, 21-31.
Klein, J.P., and Moeschberger, M.L. (1997). Survival Analysis. Techniques for Censored and Truncated Data. New York: Springer-Verlag.

Kundi, M. (1999). One-hit models for virus inactivation studies. Antivir. Res. 41, 145-152.

Kunstyr, I., and Leuenberger, H.-G.W. (1975). Gerontological data of C57BL/6J mice. I. Sex differences in survival curves. J. Gerontol. 30, 157-162.

Kurganov, B.I. (2002). Kinetics of protein aggregation. Quantitative estimation of the chaperone-like activity in test-systems based on suppression of protein aggregation. Biochemistry (Moscow), 67, 409-422.

Le Bras, H. (1976). Lois de mortalité et age limité. Population 31, 655-692.

Leeuwenburgh, C. (2003). Role of apoptosis in sarcopenia. J. Gerontol. A 58, 999-1001.

Libby, P. (2003). Bone marrow: A fountain of vascular youth? Circulation 108, 378-379.

Lindop, P.J. (1961). Growth rate, lifespan and causes of death in SAS/4 mice. Gerontologia 5, 193-208.

Lloyd, D.K. and Lipow, M. (1962). Reliability: Management, Methods, and Mathematics. Englewood Cliffs, NJ: PrenticeHall.

Makeham, W.M. (1860). On the law of mortality and the construction of annuity tables. J. Inst. Actuaries 8, 301-310.

Makeham, W.M. (1867). On the law of mortality. J.Inst. Actuaries 13, 325-358.

Martinez, D.E. (1998). Mortality patterns suggest lack of senescence in Hydra. Exp. Gerontol. 33, 217-225.

Masoro, E.J. (2003). Subfield history: caloric restriction, slowing aging, and extending life. Sci. Aging Knowledge Environ. 2003(8), RE2.

Massof, R.W., Dagnelie, G., Benzschawel, T., Palmer, R.W., and Finkelstein, D. (1990). First order dynamics of visual field loss in retinitis pigmentosa. Clin. Vision Sci. 5, 1-26.

McKiernan, S.H., Bua, E., McGorray, J., and Aiken, J. (2004). Early-onset calorie restriction conserves fiber number in aging rat skeletal muscle. FASEB J. 18, 580-581.

Mildvan, A. and Strehler, B.L. (1960). A critique of theories of mortality. In: Strehler, B.L., Ebert, J.D., Glass, H.B., and Shock, N.W. (Eds.). The Biology of Aging (pp. 216-235). Washington, DC: American Institute of Biological Sciences.

Miller, A.R. (1989). The distribution of wearout over evolved reliability structures. J. Theor. Biol. 136, 27-46.

Mueller, L., and Rose, M.R. (1996). Evolutionary theory predicts late-life mortality plateaus. Proc. Natl. Acad. Sci. USA 93, 15249-15253.

Nyengaard, J.R., and Bendtsen, T.F. (1992). Glomerular number and size in relation to age, kidney weight, and body surface in normal man. Anat. Rec. 232, 194-201.

Olshansky, S.J. (1998). On the biodemography of aging: A review essay. Population and Development Review 24, 381-393.

Olshansky, S.J., and Carnes, B.A. (1997). Ever since Gompertz. Demography 34, 1-15.

Partridge, L., and Mangel, M. (1999). Messages from mortality: The evolution of death rates in the old. Trends in Ecology and Evolution 14, 438-442.

Pearl, R., and Miner, J.R. (1935). Experimental studies on the duration of life. XIY. The comparative mortality of certain lower organisms. Quart. Rev. Biol. 10, 60-79. 


\section{Models of Systems Failure in Aging}

Peleg, M., Normand, M.D., and Campanella, O.H. (2003). Estimating microbial inactivation parameters from survival curves obtained under varying conditions-The linear case. Bull. Math. Biol. 65, 219-234.

Perks, W. (1932). On some experiments in the graduation of mortality statistics. Journal of the Institute of Actuaries 63, 12-57.

Pletcher, S.D., and Curtsinger, J.W. (1998). Mortality plateaus and the evolution of senescence: Why are old-age mortality rates so low? Evolution 52, 454-464.

Pletcher, S.D., Khazaeli, A.A., and Curtsinger, J.W. (2000). Why do life spans differ? Partitioning mean longevity differences in terms of age-specific mortality parameters. J. Gerontol. 55A, B381-B389.

Prescott, L.M., Harley, J.P., and Klein, D.A. (1996). Microbiology (3rd ed.), Dubuque, IA: WCB.

Rausand, M., and Hoyland, A. (2003). System Reliability Theory: Models, Statistical Methods, and Applications (2nd ed.), Hoboken, NJ: Wiley-Interscience.

Rauscher, F.M., Goldschmidt-Clermont, P.J., Davis, B.H., Wang, T., Gregg, D., et al. (2003). Aging, progenitor cell exhaustion, and atherosclerosis. Circulation 108, 457-63.

Ricklefs, R.E., and Scheuerlein, A. (2002). Biological implications of the Weibull and Gompertz models of aging. J. Gerontol. Ser. A 57, B69-B76.

Rigdon, S.E., and Basu, A.P. (2000). Statistical Methods for the Reliability of Repairable Systems. New York: John Wiley.

Rockstein, M., and Lieberman, H.M. (1959). A life table for the common house fly, Musca domestica. Gerontologia 3, 23-36.

Rose, M.R. (1991). The Evolutionary Biology of Aging. Oxford: Oxford University Press.

Sacher, G.A. (1966). The Gompertz transformation in the study of the injury-mortality relationship: Application to late radiation effects and ageing. In: Lindop, P.J., and Sacher, G.A. (Eds.). Radiation and Ageing (pp. 411-441). London: Taylor and Francis.

Sacher, G.A. (1977). Life table modification and life prolongation. In: Finch, C.E., and Hayflick, L., Handbook of the Biology of Aging (pp. 582-638). New York: Van Nostrand Reinhold.

Schulzer, M., Lee, C.S., Mak, E.K., Vingerhoets, F.J.G., and Calne, D.B. (1994). A mathematical model of pathogenesis in idiopathic parkinsonism. Brain 117, 509-516.

Sehl, M.E., and Yates, F.E. (2001). Kinetics of human aging: I. Rates of senescence between ages 30 and 70 years in healthy people. J. Gerontol. Ser. A 56, B198-B208.
Strasser, H., Tiefenthaler, M., Steinlechner, M., Eder, I., Bartsch, G., and Konwalinka, G. (2000). Age dependent apoptosis and loss of rhabdosphincter cells. J. Urol. 164, 1781-1785.

Strehler, B.L. (1960). Fluctuating energy demands as determinants of the death process (A parsimonious theory of the Gompertz function). In: Strehler, B.L., Ebert, J.D., Glass, H.B., and Shock, N.W. (Eds.). The Biology of Aging (pp. 309-314). Washington, DC: American Institute of Biological Sciences.

Strehler, B.L. (1978). Time, Cells, and Aging (2nd ed.). New York and London: Academic Press.

Strehler, B.L., and Mildvan, A.S. (1960). General theory of mortality and aging. Science 132, 14-21.

Strohman, R. (2002). Maneuvering in the complex path from genotype to phenotype. Science 296, 701-703.

Strohman, R. (2003). Thermodynamics-old laws in medicine and complex disease. Nature, Biotechnol. 21, 477-479.

Tatar, M., Carey, J.R., and Vaupel, J.W. (1993). Long-term cost of reproduction with and without accelerated senescence in Callosobruchus maculatus: Analysis of age-specific mortality. Evolution 47, 1302-1312.

Vanfleteren, J.R., De Vreese, A., and Braeckman, B.P. (1998). Two-parameter logistic and Weibull equations provide better fits to survival data from isogenic populations of Caenorhabditis elegans in axenic culture than does the Gompertz model. J.Gerontol. Ser. A 53, B393-B403.

Vaupel, J.W., Carey, J.R., Christensen, K., Johnson, T., Yashin, A.I., Holm, N.V., et al. (1998). Biodemographic trajectories of longevity. Science 280, 855-860.

Wallace, W.H., and Kelsey, T.W. (2004). Ovarian reserve and reproductive age may be determined from measurement of ovarian volume by transvaginal sonography. Human Reproduction 19, 1612-1617.

Weibull, W.A. (1939). A statistical theory of the strength of materials. Ingeniorsvetenskapsakademiens Handlingar $\mathrm{Nr} 151,5-45$

Weibull, W.A. (1951). A statistical distribution function of wide applicability. J. Appl. Mech. 18, 293-297.

Wilmoth, J.R. (1997). In search of limits. In: Wachter, K.W., and Finch, C.E. (Eds.), Between Zeus and the Salmon. The Biodemography of Longevity (pp. 38-64). Washington, DC: National Academy Press. 
